Difficultés de construction de savoirs et de textes problématisés en sciences de la Terre et pratiques enseignantes : étude d'une séquence ordinaire portant sur le magmatisme

Difficulties of construction of problematized knowledge and texts in Earth sciences and teaching practices: study of regular sequence concerning magmatism

\title{
Hanaà Chalak
}

\section{OpenEdition}

\section{Journals}

Édition électronique

URL : https://journals.openedition.org/educationdidactique/2063

DOI : 10.4000/educationdidactique.2063

ISSN : 2111-4838

Éditeur

Presses universitaires de Rennes

Édition imprimée

Date de publication : 20 décembre 2014

Pagination : $55-80$

ISBN : 978-2-7535-4002-6

ISSN : 1956-3485

Référence électronique

Hanaà Chalak, « Difficultés de construction de savoirs et de textes problématisés en sciences de la Terre et pratiques enseignantes : étude d'une séquence ordinaire portant sur le magmatisme », Éducation et didactique [En ligne], 8-3 I décembre 2014, mis en ligne le 20 décembre 2016, consulté le 14 septembre 2022. URL : http://journals.openedition.org/educationdidactique/2063 ; DOI : https:// doi.org/10.4000/educationdidactique.2063 


\title{
DIFFICULTÉS DE CONSTRUCTION DE SAVOIRS ET DE TEXTES PROBLÉMATISÉS EN SCIENCES DE LA TERRE ET PRATIQUES ENSEIGNANTES : ÉTUDE D'UNE SÉQUENCE ORDINAIRE PORTANT SUR LE MAGMATISME
}

\author{
Hanaà Chalak \\ Maître de conférences, Espé des Pays de la Loire
}

\begin{abstract}
À partir de l'étude des textes de savoirs (oraux et écrits) produits lors d'une séquence ordinaire (en classe de $4^{e}$ ) dans le domaine du magmatisme et de leurs relations avec les pratiques enseignantes de mise en textes, notre étude vise le repérage des difficultés d'accès à des savoirs et à des textes problématisés. Nous faisons appel à des outils empruntés au cadre théorique de la problématisation et à la théorie anthropologique du didactique (TAD) pour modéliser et mieux comprendre les pratiques de mise en texte enseignantes. Il apparaît que la mise en texte problématisé présente des difficultés en raison de techniques enseignantes centrées sur le repérage et le tri des solutions dans le but d'identifier la bonne solution au problème posé. Ces techniques semblent justifiées par des technologies marquées par les conceptions épistémologiques des enseignants et la forme habituelle des savoirs scientifiques scolaires.
\end{abstract}

Mots-clés : Problématisation, mise en texte des savoirs, pratique enseignante, séquence ordinaire, didactique des sciences de la terre.

Difficulties of construction of problematized knowledge and texts in Earth Sciences and teaching practices: study of regular sequence concerning magmatism

From the study of knowledge texts (oral and written) produced in a regular sequence (in fourth grade class) in the magmatism area and their relationship with the text-working teaching practices, our study aims to identify difficulties and didactic conditions of access to knowledge and problematized texts. We rely on both tools borrowed from the theoretical framework of problematization, the anthropological theory of didactics (ATD) to model and understand text-working teaching practices. It appears that problematized text-working presents technical difficulties due to teaching practices focused on identifying and sorting solutions in order to determine the right one for the problem. These techniques seem justified by technologies marked by the epistemological conceptions of teachers and the usual form of scientific knowledge at school.

Keywords: Problematization, knowledge text-working, teaching practices, ordinary sequence, earth science didactics. 


\section{INTRODUCTION}

La construction des savoirs problématisés en sciences fait actuellement l'objet de multiples recherches didactiques (Orange \& Orange-Ravachol, 2007, Beorchia, 2003). La préparation des jeunes à un « monde problématique » (Fabre, 2011) et la conception de l'éducation comme problématisation deviennent des nécessités de plus en plus mises en valeur par les chercheurs en sciences de l'éducation ainsi que par les programmes scolaires (ibid.). Les problèmes occupent ainsi une place remarquable dans le domaine de l'enseignement et de l'apprentissage scientifique. Cependant, certains travaux didactiques pointent la réduction des savoirs scolaires à une forme qualifiée de "propositionnelle " qui les limite à des propositions exposant des énoncés scientifiques non logiquement connectés (Astolfi, 1992, 2005). Il apparaît que les enseignants - même s'ils suivent apparemment les discours pédagogiques actuels - réduisent les savoirs à de textes simples qui dévoilent les solutions de la science sans la moindre relation aux problèmes. Le savoir présenté aux élèves est « un savoir de résultat qui ne répond à aucune question, à aucun problème » (Fabre, 2009, p. 215). Les raisons fondant les solutions scientifiques sont ignorées alors qu'une démarche problématisante les mettrait en avant. Il semble donc que le processus de construction des problèmes à l'école rencontre des difficultés qui font que la " mise en texte des savoirs » ne s'oriente pas dans le sens d'une problématisation. Nous notons que " la notion de texte peut s'appliquer à toute production verbale située, qu'elle soit écrite ou orale» (Bronckart, 1996, p. 73). La mise en texte des savoirs à l'école peut alors être définie comme étant la production de textes oraux et écrits qui fondent les savoirs dans le but d'arriver à un texte commun. D'où la nécessité, pour nous, d'étudier les pratiques enseignantes impliquées dans la construction des savoirs et des textes en classe dans le but d'identifier les difficultés d'accès aux savoirs et aux textes problématisés. Au cours de cette recherche, nous désignons, par « textes de savoir ", les productions orales et écrites mais aussi les schémas, les tableaux, les dessins d'observation etc. qui reflètent l'état des savoirs exposés au sein de la classe par les élèves et les enseignants. Avant de développer notre méthodologie de recherche, nous nous penchons sur les caractéristiques des savoirs et des textes problématisés en sciences de la Terre.

\section{CARACTÉRISTIQUES DES SAVOIRS ET DES TEXTES PROBLÉMATISÉS EN SCIENCES DE LA TERRE}

Selon Bachelard (1949), les savoirs scientifiques ne peuvent pas aucunement exister sans les problèmes avec lesquels ils maintiennent un rapport dynamique et auxquels ils sont inextricablement liés. La liaison entre le problème et le savoir accorde à ce dernier son existence et son sens. Précisément, le « savoir des réponses » ne peut prendre sens qu'en lien avec le "savoir des questions" (Fabre, 2009). Le cadre théorique de la problématisation en sciences de la vie et de la Terre (Fabre, 1999, Orange, 2000, Orange-Ravachol, 2003) au sein duquel se situe cette recherche rejoint ce positionnement épistémologique. Ce cadre postule que les savoirs scientifiques sont apodictiques c'est-à-dire organisés autour de la construction de nécessités et que l'activité scientifique est liée à des problèmes explicatifs « qui visent à expliquer des faits (phénomènes ou événements). En biologie, par exemple, il ne s'agit pas uniquement de décrire les organismes, les organes ou leurs associations, mais d'expliquer les fonctionnements à différents niveaux : écosystème, organisme, cellule, molécule...»(Orange, 2006, p. 76). De même, la géologie $^{1}$ ne se limite pas, par exemple, à la description des phénomènes volcaniques ou sismiques mais s'intéresse davantage à l'explication de leur fonctionnement. L'explication est construite à partir de la mise en relation et l'articulation de deux registres: le registre empirique, «celui des faits et des phénomènes que l'on cherche à expliquer » et le registre des modèles, "celui des élaborations explicatives construites comme tentatives de solutions » (Orange, 2003 , p. 86). Cette construction s'inspire des travaux effectués par Martinand (1986) sur la modélisation et Orange (1994) introduit un troisième registre qu'il nomme le registre explicatif. Ce dernier correspond aux " références explicatives spontanées ou habituelles du modélisateur » qui donnent le sens et le pouvoir explicatif au modèle (Orange, 2000, p. 25). La problématisation ou la mise en tension entre registre empirique et registre des modèles, sous l'organisation du registre explicatif, amène à mettre en évidence les raisons (ou les nécessités) qui contraignent les modèles explicatifs ce qui octroie un caractère apodictique au savoir. Cette valeur apodictique des savoirs scientifiques se rapproche de la définition du troisième monde de Popper (1991), celui 
des contenus objectifs de la pensée, dont les « habitants » sont les systèmes théoriques, les problèmes, les situations de problème et les arguments critiques. À partir de ces spécificités, les savoirs ne peuvent pas se limiter à des simples résultats terminaux, à des propositions factuelles et « vraies ». Ils sont porteurs des conditions de possibilités de ces solutions c'està-dire des raisons qui les sous-tendent. Le savoir scientifique problématisé ne peut se constituer que par une liaison entre le problème, sa solution, et leur engagement dans un réseau de raisons (figure 1). Par conséquent, le refoulement des raisons entraîne le renoncement au caractère apodictique des savoirs et dans ce cas-là, c'est le caractère propositionnel qui prend le devant (figure 1). D'après ces caractéristiques, un texte de savoir est considéré comme problématisé lorsqu'il garde les traces des raisons et les explicite puisque les savoirs construits ne peuvent aucunement se restreindre à la solution finale escomptée alors qu'un texte de savoir propositionnel révèle uniquement les solutions. zones bien précises du globe (dorsales océaniques, zones de subduction, points chauds) (Allègre, 1987). La fusion des roches en profondeur peut être occasionnée par des chutes de pression, des apports d'eau et elle est fonction de la composition chimique de départ. En effet, au niveau des dorsales océaniques, il apparaît que les chutes brusques de pression que subissent les matériaux ascendants avec les mouvements de convection déclenchent la fusion partielle des roches. Au niveau des zones de subduction, les conditions sont totalement différentes «puisque les matériaux qui fondent ne remontent pas vers la surface mais au contraire s'enfoncent vers l'intérieur, vers les hautes pressions» (ibib. p. 110). Le réchauffement de la plaque froide qui s'enfonce, libère l'eau gorgée dans la croûte océanique par des réactions métamorphiques ce qui entraîne la fusion des péridotites de la plaque chevauchante. Ce type de présentation des savoirs est factuelle, assertorique, se situe dans l'ordre épistémologique du vrai et du faux et ne présente aucun caractère de nécessité puisqu'il

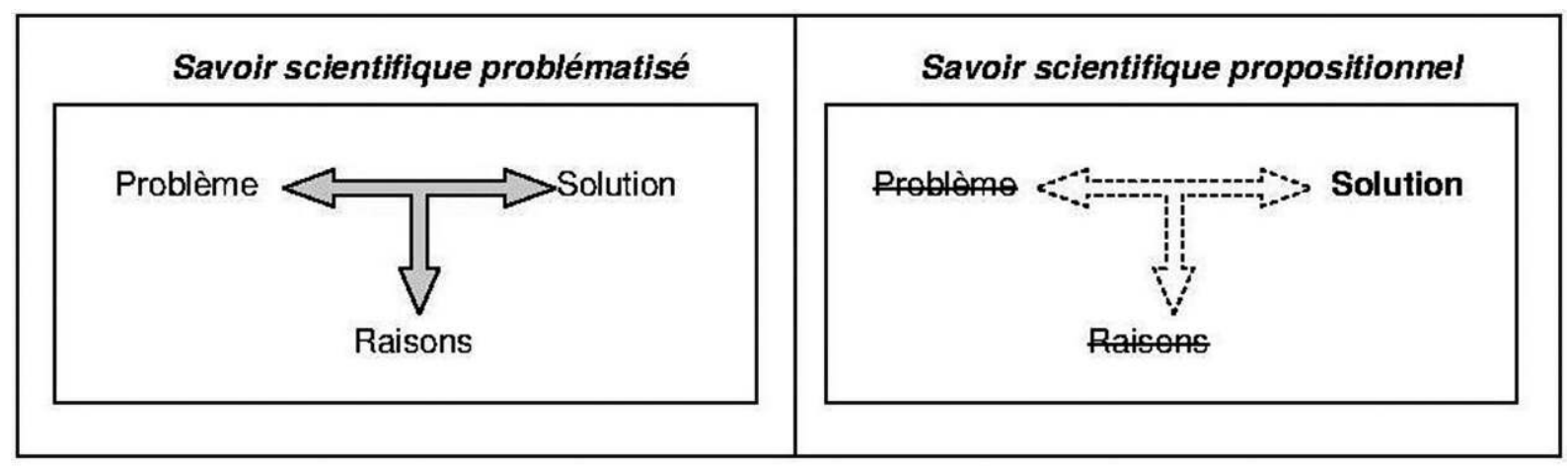

Figure 1. Relation entre le problème, sa solution et les raisons dans un savoir problématisé et dans un savoir propositionnel (Chalak, 2012a).

Exemple du problème du magmatisme

Afin de distinguer une focalisation sur les solutions d'une focalisation sur les nécessités qui contraignent ces solutions, nous présentons l'exemple du problème du magmatisme. Accorder de l'importance à la solution de ce problème, dans sa globalité, consiste à expliquer la fabrication du magma par des fusions partielles du manteau solide se déroulant sous des conditions particulières, en relation avec la tectonique des plaques, et dans des s'agit de « savoir que c'est comme cela». Un texte de savoir propositionnel en sciences de la Terre se limite à exposer ces solutions. La transmission des savoirs aux élèves sous une forme propositionnelle véhicule une vision simpliste de la science comme une accumulation de faits ne soulevant aucun problème. Dans une approche de problématisation, il est important que les élèves puissent passer, à travers la mise en place de situations comme les débats scientifiques, d'une problématique de sortie d'un magma liquide préexistant à l'intérieur de la Terre à une problématique de fabrication de magma à des conditions précises (Orange \& Orange-Ravachol, 2004). De plus, il est important qu'ils intègrent les conditions de possibilités de cette problématique (nécessité d'une production locale du magma et nécessité de 
conditions locales permettant la fusion) et qu'ils soient capables d'expliquer pourquoi cette formation est possible et nécessaire et pourquoi il ne peut pas en être autrement (Reboul, 1992). Précisément, l'explication de la formation du magma au niveau des zones de subduction, par exemple, « convoque et articule des contraintes empiriques (le manteau solide, des données expérimentales sur la fusion des roches, etc.) et des contraintes théoriques inhérentes au cadre de la tectonique des plaques (le concept de plaques, de zone de subduction, etc.) jusqu'au point d'établir la nécessité d'une production locale de magma à une certaine profondeur à l'aplomb des volcans des zones de subduction, et celle d'un mécanisme capable d'abaisser la température de fusion des roches mantelliques » (Orange-Ravachol, 2010, p. 49). Un texte de savoir serait qualifié de problématisé dans la mesure où il garde les traces de ces raisons en plus des solutions. Par ailleurs, il semble que la textualité des savoirs scolaires induit certaines difficultés à l'école.

\section{DIFFICULTÉS INDUITES PAR LA FORME TEXTUELLE DES SAVOIRS SCOLAIRES}

La transposition didactique, permettant le passage du savoir savant au savoir scolaire, suppose « la production d'un texte du savoir » (Chevallard, 1991, p. 65) qui rend ce dernier accessible aux élèves. L'enseignement scientifique, tout comme la pratique scientifique, débouche sur des textes. Ce processus de sélection des éléments de la culture et de leur mise en texte, qui vise leur préparation à être transmis à l'école, ne peut pas être évité et n'est pas sans incidence sur les savoirs. En effet, la mise en texte des savoirs scolaires est un processus d' " apprêt » didactique (Chevallard, 1991) qui serait à l'origine des contraintes signalées par Verret (1975). Cet auteur (ibid., p. 146-147) établit que la « transmission bureaucratique du savoir » provoque sa dépersonnalisation, sa désyncrétisation, et une programmation de son acquisition. Elle requiert quant à la transmission « la publicité du savoir » c'est-à-dire sa définition explicite et « le contrôle social des apprentissages ». Dans le milieu scolaire, le rôle des enseignants serait de présenter ces savoirs, déterminés par les programmes scolaires, aux élèves à travers un ensemble de techniques scolaires (énoncésproblèmes, construction d'exercices, etc.) (Brossard,
1998). Ils vont les emmener, via ces techniques, à mettre en texte les savoirs scientifiques c'est-à-dire à produire des textes oraux et écrits dans l'objectif de parvenir à un texte de savoir commun. Rey (2005, p. 99) voit que la forme scolaire des apprentissages intervient dans le type de problématisation attendu des élèves par l'école : «La textualité est un des principes régulateurs des problématisations scolaires. » Cet auteur considère que l'école valorise une certaine forme de problématisation régie par une exigence de textualité. Les caractères retenus et isolés d'une situation sont ceux qui s'accommodent à l'économie du texte. L'obligation de «textualité » des savoirs structure les pratiques enseignantes et induit certaines complications et difficultés chez les élèves. En effet, même si les enseignants suivent les discours pédagogiques actuels qui les exhortent à engager les élèves dans des tâches et des problèmes, le « savoirtexte » n'est pas pour autant éliminé (Rey, 2007). Au contraire, il reste omniprésent et cette mise en activité finit par aboutir à ce « savoir-texte» final. Cette manière de faire, au lieu de confronter les élèves d'une manière directe aux textes de savoirs, enrôle ceux-ci dans des activités, des problèmes, des tâches, des situations-problèmes mais n'élimine pas toute référence au savoir textuel et fait ainsi des activités un moyen d'accès à ce savoir qui constitue le but et l'intention ultime. La " tâche " scolaire, y compris quand elle consiste en une construction de problème, n'est donc pas là pour remplacer le savoir textuel, mais comme un moyen pour y " accéder » (Rey, 2007 , p. 177). Kahn \& Rey (2008, p. 22) établissent que certains élèves éprouvent des difficultés dès lors qu'il s'agit de « tirer de la situation singulière de la classe, les outils de savoir reconstruits ou utilisés par les élèves dans l'activité et d'en extraire et consigner le caractère universel, autrement dit : mettre en texte le savoir $»$. Plus spécifiquement, le passage d'une parole, dont le sens s'élucide par le renvoi aux réalités extérieures, à des énoncés particuliers d'un texte dont le sens est tiré du rapport réciproque entre ces énoncés au sein du texte et à des références existant dans d'autres textes non présents mais partagé par le groupe, semble engendrer ces difficultés. La focalisation des élèves reste attirée par les aspects réels de l'activité dont ils ne trouvent qu'un moyen pour en tirer les conséquences, la raconter. Ils restent prisonniers des particularités de l'activité qu'ils viennent de réaliser ce qui entrave leur saisie du savoir en jeu et leur perception du sens de l'activité 
au-delà du contexte de travail. Ceci nous conforte dans le choix d'étudier la manière dont se fait cette mise en texte en sciences de la Terre et les difficultés qu'elle rencontre.

\section{MÉTHOdOLOGIE DE RECUEIL ET D'ANALYSE DES DONNÉES}

\author{
Une séquence ordinaire comme données \\ recueillies
}

Pour étudier les difficultés d'une mise en texte problématisé en sciences de la Terre, nous nous intéressons à une séquence ordinaire portant sur le problème de l'origine des matériaux volcaniques en $4^{\mathrm{e}}$. Les séquences ordinaires sont dispensées en classe sous l'intégrale responsabilité de l'enseignant. Elles se font sans aucune intervention ni aide dans le choix des enseignements de la part des chercheurs qui sont de simples observateurs de ce qui se passe habituellement en classe. Ces derniers s'abstiennent d'influencer ce qu'ils observent et ne cherchent, en aucun cas, à modifier les effets attendus. L'étude de ces situations s'avère essentielle pour « rendre compte du travail des élèves et des professeurs, dans les classes ordinaires » (Mercier, 2008). C'est pour cela que ces dernières années, les recherches en didactique des disciplines portant sur l'analyse de ces situations, se sont multipliées (Venturini, Calmettes, Amade-Escot \& Terrisse, 2007 ; Calmettes, 2011). Nous nous intéressons à ce type de séquence parce que c'est le travail ordinaire de mise en texte des élèves et de leurs enseignants tel qu'il se fait en classe que nous voulons étudier. La séquence, qui constitue notre corpus d'étude, est composée de quatre séances et se déroule dans une classe de $4^{\mathrm{e}}$ de vingt élèves âgés de 12 à 14 ans. Elle s'intègre dans la partie du programme consacrée à l'étude de l' " activité interne du globe » (M.E.N., 2008, p. 47). Les objectifs d'apprentissage mentionnés dans les programmes officiels sont les suivants : " Les magmas sont contenus dans des réservoirs magmatiques localisés, à plusieurs kilomètres de profondeur » (ibid., p. 48). Les phénomènes magmatiques, si l'on s'appuie sur ces recommandations, sont envisagés sans lien avec la dynamique du globe et la tectonique des plaques traitées ultérieurement selon la progression de cette partie du programme de $4^{\mathrm{e}}$. Nous disposons des enregistrements vidéo et audio de l'ensemble des séances (1-4), de leur transcription, du recueil des productions écrites individuelles et de groupe élaborées par les élèves tout au long de la séquence. L'enseignante est expérimentée et participe aux recherches d'un groupe de travail qui s'intéresse à la construction des savoirs problématisés en sciences et à ses conditions. Vu son statut, il nous est apparu intéressant d'étudier les pratiques ordinaires qu'elle mobilise dans la construction des savoirs dans le domaine du magmatisme au sein de sa classe et la place de la problématisation qu'elle y accorde.

\section{Analyse de la séquence étudiée}

La séquence a été analysée selon une méthodologie que nous avons mise en œuvre pour répondre à nos questions de recherche (figure 2). D'abord, il s'agit de décrire et d'analyser la séquence didactique. Pour cela, nous avons fait appel à la notion de synopsis qui « correspond à la première analyse que fait le chercheur quand il travaille avec les données correspondant à une séance [...]. Dans cette première analyse, le chercheur se situe en observateur extérieur; il prend le point de vue global de la classe $[\ldots] »$ (Tiberghien, Malkoun, Buty, Souassy \& Mortimer, 2007, p. 105)². L'étape suivante consiste à caractériser les textes produits lors de la séquence, les comparer et étudier leur contenu du point de vue de leur relation au problème posé et à la problématisation : les textes portent-ils des traces de la problématisation ? Sont-ils propositionnels ? Quelle est leur fonction au sein de la séquence? Quels liens entretiennent-ils les uns avec les autres? Finalement, nous remontons aux pratiques ayant conduit à la production de ces textes : comment ces textes ont-ils été construits? Qui des élèves ou de l'enseignant les construisent? Quelles pratiques ont-elles servi leur construction? (figure 2). Cette dernière analyse se fera en deux temps :

- Lanalyse des textes nous permettra de modéliser les pratiques enseignantes de mise en texte grâce à des outils empruntés à la théorie anthropologique du didactique (TAD) introduite en didactique des mathématiques par Chevallard $(1997,1998)$. Nous portons particulièrement notre attention à la notion de praxéologie ou d'organisation praxéologique qui s'intéresse à la façon dont un être humain, au sein d'une institution, résout une tâche donnée. Cette notion vise à analyser toute action 
humaine en termes de bloc pratico-technique (savoir-faire) et de bloc technologico-théorique (savoir). «En toute institution, l'activité des personnes occupant une position donnée se décline en différents types de tâches T, accomplies au moyen d'une certaine manière de faire, ou technique, t. Le couple [T/t] constitue, par définition, un savoir-faire. Mais un tel savoirfaire ne saurait vivre à l'état isolé : il appelle un environnement technologico-théorique [q/Q], ou savoir (au sens restreint), formé d'une technologie, q, "discours" rationnel (logos) censé justifier et rendre intelligible la technique (tekhnê), et à son tour justifié et éclairé par une théorie, Q, généralement évanouissante * (Chevallard, 1997, p. 37). De plus, une théorie peut justifier un ensemble de technologies dont chacune à son tour justifie et rend intelligibles plusieurs techniques correspondant à autant de types de tâches (Chevallard, 1996). À partir de ces définitions, Bosch et Chevallard (1999) considèrent qu'une organisation praxéologique ou praxéologie est formée du complexe de techniques, de technologies et de théories qui permettent d'accomplir un type de tâches. Le schéma technique/technologie/ théorie vaut pour toutes les activités humaines (Chevallard, 1996). Par ailleurs, « ce qui à un moment donné ou dans une institution donnée, apparaît comme la justification d'une certaine technique, peut aussi être considéré, ailleurs ou à un autre moment, comme une tâche en elle-même (la tâche consistant à justifier une technique), qui suppose la mise en œuvre d'une technique particulière et l'élaboration d'un environnement technologico-théorique adéquat » (Bosch \& Chevallard, 1999, p. 84). Ainsi, le couple technique/technologie/théorie doit toujours être référé au type de tâches que l'on prend en considération. Bosch et Chevallard (1999, p. 92) notent également que « la mise en ouvre d'une technique se traduit par une manipulation d'ostensifs réglée par des non-ostensifs ». Les ostensifs représentent la partie "matérielle » de l'activité qui se donne à voir lors de la réalisation de la tâche comme les mots, discours, écritures, symboles, graphismes et gestes (Bosch \& Chevallard, 1999). Ils constituent les ingrédients des tâches, techniques, technologies et théories. Quant aux objets non ostensifs, ils constituent les concepts, les idées, les notions, etc.

Dans notre recherche, nous considérons que la mise en texte des savoirs scientifiques relève d'un type de tâche enseignant dont l'accomplissement appelle des techniques spécifiques justifiées par des technologies puis des théories que notre étude se propose d'identifier. Les notions de la TAD sont retravaillées dans une perspective comparatiste (Mercier, Schubauer-Leoni \& Sensevy, 2002) en s'appuyant sur la distinction générique/spécifique. Celle-ci nous emmène à désigner ce qui est générique au didactique et spécifique à la didactique des sciences de la Terre. Cette approche comparatiste a été mobilisée par une étude menée par Santini (2010) concernant les systèmes sémiotiques (la coupe d'un appareil volcanique) dans l'action conjointe en didactique.

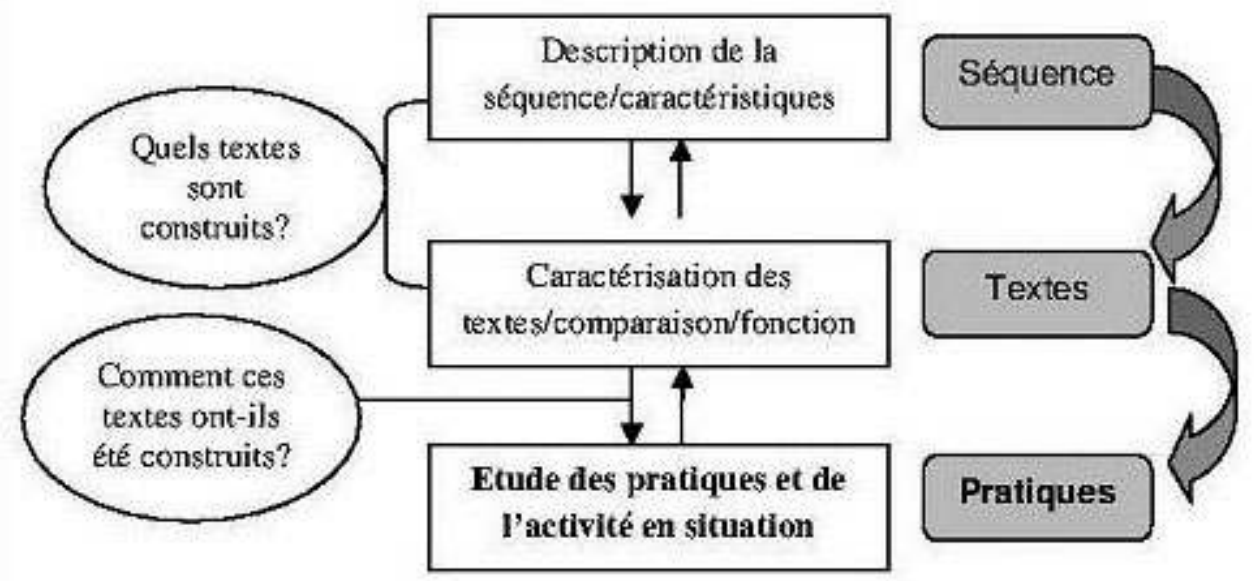

Figure 2. Méthodologie générale d'analyse de la séquence ordinaire de notre recherche. 
- Nous étudions les pratiques ayant participé à la construction de ces textes. Nous portons notre attention à l'activité des enseignants en interaction avec celle des élèves au sein des situations d'enseignement-apprentissage. À ce stade, ce sont les discussions disponibles via les enregistrements et les transcriptions effectuées qui constituent les supports des analyses. Le cadre théorique de la problématisation sera utilisé pour l'étude des interactions élève-enseignant et la TAD pour l'analyse de l'action enseignante seule.

Nous notons que les niveaux d'analyse ne sont pas isolés et le dispositif méthodologique construit envisage la possibilité de rétroaction d'un niveau sur l'autre (Schubauer-Leoni \& Leutenegger, 2002) (figure 2). Après la présentation de la méthodologie de recueil et d'analyse des données, nous démarrons l'étude de la séquence ordinaire selon les repères définis.

\section{ÉTUDE DE LA MISE EN TEXTE DES SAVOIRS ET DES PRATIQUES ENSEIGNANTES DANS UNE SÉQUENCE ORDINAIRE}

Nous commençons par décrire la séquence telle qu'elle s'est déroulée pour repérer ses caractéristiques générales puis nous entamons l'analyse des écrits et des pratiques de mise en texte.

\section{Description et organisation de la séquence ordinaire}

Nous présentons une description détaillée de la séquence ordinaire de notre recherche en précisant les écrits produits :

\section{Séance 1 et 2}

Individuellement, les élèves répondent à la question : "Propose un dessin très simple d'un volcan en coupe où tu places le lieu d'où viennent les matériaux, avec des hauteurs possibles, avec l'emplacement du sol par rapport à ces matériaux etc. À légender. » Puis, par groupes de trois ou cinq ils confrontent leurs écrits individuels pour proposer un modèle commun sous forme de schéma légendé. Ce travail est suivi d'un débat ${ }^{3}$ portant sur les modèles élaborés par les groupes. Le débat aboutit à l'identification de trois propositions concernant l'origine des matériaux volcaniques et conduit à la production d'un premier écrit « intermédiaire $»^{4}$ E1 (figure 3) si nous reprenons les termes de Chabanne et Bucheton (2002) :

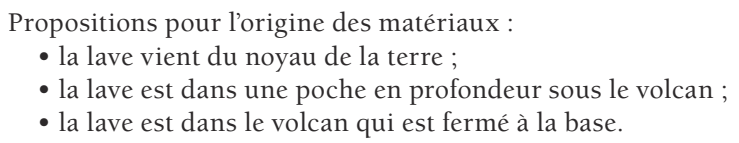

Figure 3. Résultat du débat scientifique (E1).

\section{Séance 3}

Après un rappel des trois propositions repérées antérieurement, les élèves exploitent collectivement un document scientifique montrant l'enregistrement de l'activité sismique sous un volcan (annexe 1) ce qui aboutit à la validation de la deuxième proposition : « La lave est dans une poche en profondeur sous le volcan. » Les deux autres propositions sont invalidées à l'issue de discussions orales. La séance prend fin avec l'élaboration d'un deuxième écrit intermédiaire E2 (figure 4) et d'un écrit bilan final E3 (figure 5):

\footnotetext{
Résultat : documentation scientifique.

On observe des séismes alignés sous le volcan mais en deux groupes séparés par une zone sans séismes. Cette zone est une zone liquide (où il n'y a pas de ruptures, donc pas de foyers sismiques). Elle correspond, probablement, à un réservoir de magma.

Peut-il être alimenté par le noyau de la terre?

Plus on descend en profondeur dans la terre, plus la température augmente, ce qui pourrait permettre de rendre liquide la roche, mais en même temps, la pression augmente, et rend le noyau complètement solide.
}

Figure 4. Résultat de la confrontation avec la documentation scientifique (E2).

\footnotetext{
Origine de ces manifestations

Activité 2 : D'où viennent les matériaux ?

- La lave provient d'un magma contenu dans un réservoir (qui peut être situé jusqu'à $70 \mathrm{~km}$ de profondeur).

- Le magma est de la roche fondue contenant des gaz, et des morceaux de roches solides. La lave ne contient plus les gaz.
}

Figure 5. Écrit bilan final sur l'origine des manifestations de l'activité volcanique (E3). 


\section{Séance 4}

Un schéma bilan final E4 (figure 6$)^{5}$ représentant une coupe simplifiée d'un volcan actif est construit au tableau et recopié par les élèves. La construction de ce schéma est accompagnée d'une réflexion collective sur le moteur qui emmène les matériaux à la surface. Le texte concernant la remontée des matériaux est noté sous le schéma bilan.

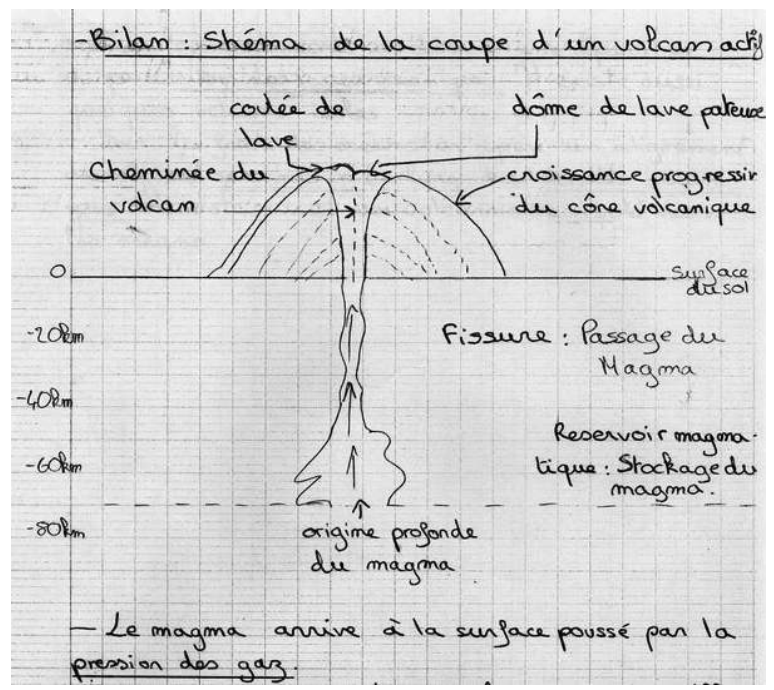

Figure 6. Schéma bilan final de la coupe d'un volcan actif ${ }^{6}$ (E4).

\section{Caractéristiques générales de la séquence étudiée}

Afin de déterminer les principales caractéristiques de cette séquence et d'en réaliser une analyse globale, nous avons construit un synopsis (tableau 1) qui donne à voir des dimensions aménagées en correspondance avec nos questions de recherche. Son analyse fait nettement apparaître une variété dans les écrits portant à la fois sur leur mode de production (individuel, groupe, classe) et sur leur forme (texte, schéma). Les écrits initiaux (explications individuelles et de groupe) autorisent l'expression des idées explicatives des élèves. Nous pouvons les considérer comme étant des « écrits d'investigation » qui mobilisent la pensée des élèves et les aident à problématiser. Le rôle précis des écrits intermédiaires et finaux (E1, E2, E3, et E4) sera élucidé dans la partie suivante. À ces différents écrits sont associées des productions langagières orales (discussions, débat). Les fonctions de ces activités langagières qui jalonnent la séquence étudiée sont primordiales. En effet, « [...] les écrits servent à décrire, à expliquer, à prévoir ; les échanges oraux permettent la critique, mais aussi la négociation, l'ajustement du travail en commun » (Orange, 2009, p. 245). Quant à l'organisation de la classe, elle passe de l'individuel au collectif avec l'avancée de la séquence. De plus, les textes sont réalisés au début par les élèves individuellement puis en groupe tandis que les quatre écrits suivants (E1, E2, E3, E4) sont l'œuvre de l'enseignante.

À première vue, la procédure suivie exprime une volonté de l'enseignante de s'inscrire dans une démarche de problématisation. La mise en œuvre d'un débat scientifique basé sur l'expression des explications initiales des élèves et de leurs idées puis la confrontation avec la documentation scientifique laissent penser que cette séquence a été conçue dans le but que les élèves accèdent à des savoirs problématisés. Ceci s'explique par la réflexion théorique que mène l'enseignante en question avec un groupe de recherche qui se penche sur le processus de problématisation et sur l'importance des pratiques langagières dans les apprentissages. Bien évidemment, l'expression de cette volonté ne conditionne pas forcément l'implication des élèves dans la construction des savoirs problématisés. Pour cela, nous nous proposons d'analyser cette construction en commençant par les écrits produits lors de la séquence et en remontant par la suite aux pratiques ayant permis leur mise en place. 


\begin{tabular}{|c|c|c|c|c|c|}
\hline Séances & Organisation & Productions langagières & Forme des & Nature des & Auteur des \\
\hline & & & & & \\
\hline \multirow{3}{*}{1 et 2} & Individuelle & $\begin{array}{l}\text { Ecrits individuels - Productions } \\
\text { explicatives }\end{array}$ & $\begin{array}{l}\text { Schéma } \\
\text { légendé }\end{array}$ & \multirow[b]{2}{*}{ Eccrits initiaux } & Élève \\
\hline & En groupe & $\begin{array}{l}\text { Echanges oraux, discussions de } \\
\text { groupe } \\
\text { Ecrits de groupe - Productions } \\
\text { explicatives }\end{array}$ & $\begin{array}{l}\text { Schéma } \\
\text { légendé }\end{array}$ & & $\begin{array}{l}\text { Groupe } \\
\text { d'élèves }\end{array}$ \\
\hline & Collective & $\begin{array}{l}\text { Échanges oraux, débat collectif } \\
\text { E1 : Résultat du đébat scientifique }\end{array}$ & Texte & \multirow{2}{*}{$\begin{array}{c}\text { Écrits } \\
\text { intermédiaires }\end{array}$} & \multirow{4}{*}{$\begin{array}{l}\text { Écrits de } \\
\text { l'enseignante } \\
\text { synthétisant } \\
\text { les échanges } \\
\text { oraux }\end{array}$} \\
\hline \multirow[t]{2}{*}{3} & \multirow[t]{2}{*}{ Collective } & \multirow{2}{*}{$\begin{array}{l}\text { Echanges oraux, discussion } \\
\text { collective } \\
\text { Ecrit } 2 \text { : Résultat de la } \\
\text { confrontation avec la } \\
\text { docum entation scientifique } \\
\text { Écrit } 3: \text { Écrit bilan final }\end{array}$} & Texte & & \\
\hline & & & Texte & \multirow{2}{*}{$\dot{E}$ crits finaux } & \\
\hline 4 & Collective & $\begin{array}{l}\text { Échanges oraux collectifs } \\
\text { Écrit } 4 \text { : Schéma bilan final de la } \\
\text { coupe d'un volcan actif }\end{array}$ & $\begin{array}{l}\text { Schéma } \\
\text { légendé } \\
\text { accompagné } \\
\text { d'un texte }\end{array}$ & & \\
\hline
\end{tabular}

Tableau 1. Synopsis de l'organisation de la séquence ordinaire de $4^{e}$ et des activités langagières mises en oeuvre.

Analyse des différents écrits élaborés au cours de la séquence

Nos questions et nos objectifs de recherche nous emmènent à porter notre attention sur les différents écrits élaborés pendant la séquence. Ainsi, après avoir identifié leur forme, leur nature et leur place dans la situation étudiée, il s'agit d'analyser leur contenu en nous focalisant sur ce qu'ils évoquent à propos de l'origine des matériaux volcaniques et le fonctionnement des volcans. Les écrits individuels et de groupe sont des écrits initiaux d'élèves, le premier écrit intermédiaire E1 clôture le débat, le deuxième E2 la phase d'exploitation de la documentation scientifique et les écrits E3 et E4 correspondent à des bilans terminaux. Nous examinons le contenu de ces textes pour savoir s'ils portent les traces de la problématisation ou s'ils se contentent d'exposer les solutions sous une forme propositionnelle. Leurs fonctions et les liens qu'ils entretiennent les uns avec les autres seront élucidés.

- Les écrits explicatifs initiaux sont constitués d'un schéma légendé et réalisés individuellement (20 productions) puis en groupe
( 5 productions) par les élèves de la classe de $4^{\mathrm{e}}$. Leur analyse permet d'inférer les conceptions des élèves (Santini, 2009) et de révéler comment ceux-ci expliquent spontanément l'origine des matériaux volcaniques. Les modèles des élèves traduisent une certaine hétérogénéité d'un point de vue fonctionnel et révèlent plusieurs types d'explications ;

- Certains écrits situent l'origine des matériaux au niveau d'un environnement local, se limitant au volcan lui-même ( 8 productions individuelles (PI) sur 20 et 2 productions de groupe (PG) sur 5). L'origine de la lave est superficielle : soit elle remplit le volcan (7 PI sur 20 et 1 PG sur 5) (figure 7), soit elle est contenue dans un réservoir fermé situé dans le volcan (1 PI sur 20 et 1 PG sur 5). Ainsi, le volcanisme est expliqué sans avoir besoin à le situer dans un environnement large en référence à la planète Terre. C'est une forme d'explication simple qui correspond à la « conception locale » du magmatisme selon Orange (1995). Toutefois, il reste difficile de savoir à ce stade si, pour les élèves, cette lave préexiste dans le volcan ou si elle y est fabriquée. De plus, les propos avancés pendant le 
débat scientifique sur les affiches de groupe concernées ne nous ont pas tellement aidés pour accéder à cette information.

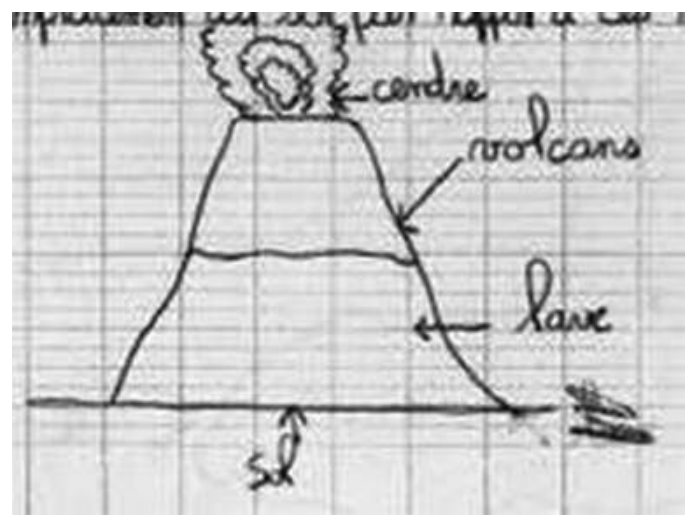

Figure 7. Exemple d'écrit initial individuel avec une origine des matériaux volcaniques située au niveau d'un environnement local.

- Plusieurs productions dévoilent un réservoir situé dans le "sous-sol ", sous terre (6 PI sur 20 et 1 PG sur 5) (figure 8) ou un magma qui remplit ce sous-sol (2 PI sur 20). Ces écrits indiquent un début de référence à la Terre. Pour ceux réalisés individuellement, il s'avère compliqué d'évaluer le degré de cette référence à la Terre. Quant à la production du deuxième groupe (figure 8), les élèves indiquent au cours du débat que la poche n'est pas très en profondeur.

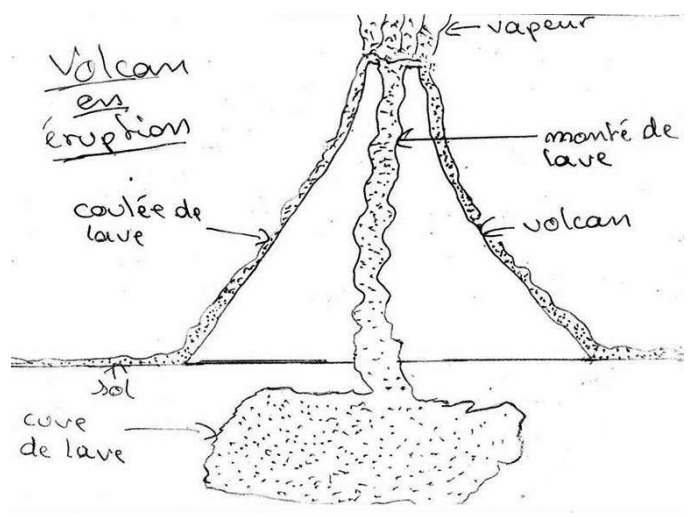

Figure 8. Exemple d'écrit initial de groupe avec un réservoir de magma situé sous la surface du sol.

- D'autres voient le volcanisme comme un phénomène se produisant dans un environnement large relié aux caractéristiques de la Terre qui renfermerait à l'intérieur - spécifi- quement dans le noyau - les matériaux volcaniques sous forme liquide (1 PI sur 20 et 1 PG sur 5). L'explication du groupe concerné indique, lors du débat, que le noyau représente un endroit où se trouve la lave : « 2. Amandine. Hum on a représenté donc le volcan et puis ben dans la Terre y a un noyau où y a des phénomènes comme euh la lave etc. (rire) et euh ben c'est là où euh, où la lave elle vient, elle vient au volcan » (figure 9). Cette explication correspond à une « conception centrale » du magmatisme (Orange, 1995).

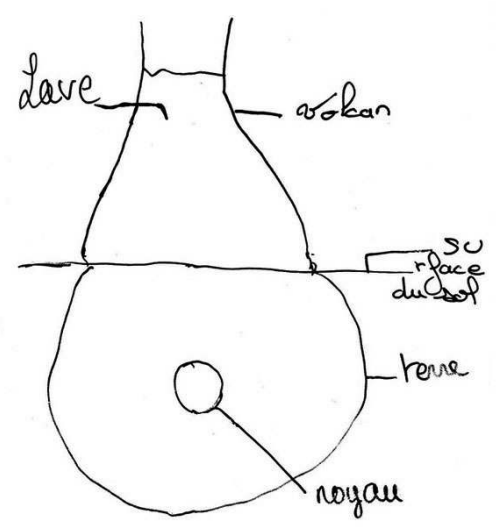

Figure 9. Exemple d'écrit initial de groupe avec un magma ayant pour origine le noyau terrestre.

Ces analyses montrent que les explications des élèves sur le fonctionnement des volcans sont hétérogènes. Certaines productions révèlent un fonctionnement local limité à l'environnement du volcan, d'autres se rapportent à une explication centrale reliant le volcanisme à l'activité du globe terrestre. Toutefois, il nous a été difficile d'évaluer pour quelques productions le degré de référence à la Terre. Par ailleurs, nous notons l'absence de référence aux plaques tectoniques et à une production de magma reliée à des endroits précis de la planète comme le suggère la " conception globale » (Orange, 1995) actuelle du magmatisme. Étant donné que chacun de ces écrits donne à voir une solution, du point de vue des élèves, au problème posé nous considérons qu'il s'agit de «mono-textes propositionnels». Les affiches de groupe ont servi comme support pour le débat de classe qui a été suivi par la production de l'écrit intermédiaire El. 
- Écrit 1 : Cet écrit intermédiaire correspond à une liste de trois solutions distinctes au problème de l'origine des matériaux volcaniques: la lave existe soit au niveau du noyau, soit au niveau d'une poche pas très profonde ou alors dans le volcan lui-même. Ces solutions mettent en avant une lave qui préexiste déjà dans les endroits mentionnés à l'état liquide et ne posent pas la question de son mode de formation. Rappelons que les géologues actuels se penchent plutôt sur le problème de la formation du magma à certains endroits et pas à d'autres en lien avec certains phénomènes tectoniques (Orange-Ravachol, 2003). La principale fonction de ce texte est de repérer les différentes thèses proposées et nous y retrouvons les principales explications des élèves révélées lors de l'étude précédente de leurs productions individuelles et de groupe. $\mathrm{Au}$ regard de notre cadre didactique de la problématisation, cet écrit propose plusieurs possibles et contribue donc à l'exploration des possibles. Toutefois, il se focalise sur les solutions et n'explicite en aucun cas les raisons qui fondent celles-ci. Puisque l'écrit E1 reflète des points de vue divergents en présentant plusieurs thèses pour l'explication du problème posé sans évoquer les raisons, nous le nommons «pluri-texte propositionnel».

Écrit 2 : Le deuxième écrit intermédiaire E2 succède à l'exploitation collective d'un document scientifique montrant l'enregistrement de l'activité sismique sous un volcan (annexe 1) et présente plusieurs niveaux. D'abord, il s'agit de décrire le document en se basant sur un constat empirique puis d'emmener une explication argumentée à l'état liquide de la zone sans séismes observée sous le volcan. Ainsi, l'absence de foyers sismiques au niveau d'une zone particulière sous le volcan, suggère la présence d'une zone fluide qui correspond à un réservoir de magma. Ceci apporte des argumentations en faveur de la deuxième proposition de l'écrit El qui signale que la lave provient d'une poche non profonde. La seconde partie de l'écrit E2 argumente l'impossibilité d'une origine de la lave située au centre de la Terre puisque celui-ci est solide contrairement à ce que les élèves ont pu penser. Ces arguments sont avancés contre la première thèse de l'écrit El. Toutefois, aucun argument n'est mentionné pour invalider la troisième proposition. La principale fonction de l'écrit E2 est de valider et d'invalider deux propositions repérées précédemment dans l'écrit E1. Il permet de trier les thèses déjà relevées et d'apporter des argumentations qui favorisent ou défavorisent l'une ou l'autre. Lécrit E2 est un « mono-texte argumentatif » car il présente plusieurs argumentations compatibles participant au tri des solutions.

Écrit 3 : Lécrit bilan final E3 est élaboré directement après l'écrit E2 et comprend deux parties. La première expose la bonne solution au problème posé. La seconde précise la différence entre le magma et la lave. Du point de vue de la problématisation, ce texte ne fait que présenter la solution retenue au problème géologique posé. Les raisons qui fondent ces solutions ne sont pas explicitées. De plus, la solution proposée relève plusieurs ambigüités par rapport aux explications scientifiques actuelles. Ainsi, on pourrait croire que c'est le réservoir qui est à l'origine du magma et qu'il est fermé à la base alors qu'il ne sert que pour son stockage. Rien n'est évoqué, dans cet écrit E3, sur le mode de formation de ce magma ni sur l'endroit et les conditions de sa formation. La focalisation sur un « magma préexistant » fait que les problèmes de la production du magma et de son renouvellement ne se posent pas au contraire de celui de son lieu d'existence. Ce texte donne à voir des caractéristiques qui l'éloignent d'un texte problématisé pour le rapprocher plutôt d'un texte propositionnel. Si nous le comparons avec le texte E2 précédent, nous constatons que ce dernier porte des argumentations permettant de valider la solution finale et d'invalider les autres mais qui ont disparu avec l'écrit final E3. C'est un « mono-texte propositionnel » qui présente la solution finale au problème tout en rajoutant des précisions concernant la différence entre le magma et la lave. Le magma étant de la roche fondue contenant des gaz et des morceaux de roches solides tandis que la lave ne contient plus les gaz. Ceci sous-entend que le magma s'est formé suite à la fusion de la roche solide située en profondeur sachant qu'il n'a jamais été question de fusion dans l'explication proposée pour l'origine des matériaux volcaniques.

Écrit 4 : Le schéma bilan E4 (figure 6) correspond à un schéma bilan final légendé de la coupe d'un volcan actif, accompagné d'un texte. Ce schéma explicatif mentionne le stockage du magma, à origine profonde, au niveau d'un réservoir magmatique situé à - $70 \mathrm{~km}$ de 
profondeur et révèle l'existence d'une fissure permettant le passage du magma de ce réservoir vers la surface terrestre. Cette origine profonde du magma n'apparaissait pas dans l'écrit E3 précédent. Toutefois, rien n'est précisé sur cette profondeur: est-ce une origine très profonde ou peu profonde, est-ce au niveau du manteau, du noyau? Le problème de la formation du magma n'est pas posé ici non plus. Celui-ci est stocké dans le réservoir et ne demande qu'à remonter. Notons que le magma progresse rarement par « une fissure " lors de sa remontée mais par un réseau de fissures. De plus, l'écrit E4 précise que l'arrivée du magma à la surface se fait sous la pression des gaz mais rien ne rend cette remontée nécessaire pour le fonctionnement du volcan. Par ailleurs, les recherches qui se sont intéressées aux conceptions des élèves en rapport avec le volcanisme (Orange, 1995 ; Goix, 1995) indiquent que les élèves de la classe de $4^{\mathrm{e}}$ ont souvent tendance à penser que le magma existe sous forme liquide et qu'il provient des profondeurs terrestres. Le schéma bilan produit risque d'appuyer ces idées même si l'écrit intermédiaire E2 avance des argumentations qui soutiennent l'impossibilité d'un magma provenant du noyau car celui-ci est solide. Comme l'écrit E3 précédent, c'est un « monotexte propositionnel ». Ainsi, les écrits E3 et E4 finaux présentent des caractéristiques qui les éloignent de celles d'un écrit problématisé. Ce dernier mettrait en avant les raisons (nécessité de la formation du magma et nécessité de sa remontée) en plus des solutions. Le tableau 2 ci-dessous présente un exemple de ce que pourrait être un écrit final problématisé concernant le magmatisme en classe de $4^{\mathrm{e}}$.

Comment est-ce dans la Terre pour que ça marche?

Il faut que le magma remonte Il faut que le magma se forme

Le magma est poussé par la La roche fond à des profonpression des gaz. Cela crée deurs de la Terre (100 à des failles et des cheminées $200 \mathrm{~km}$ ) à des températures pour que le magma remonte. d'environ $1000^{\circ}$ à $2000^{\circ}$ et Son lieu de fabrication peu sous une certaine pression. profond par rapport à l'échelle Cela se forme à environ -70 de la Terre permet sa remon- à $-200 \mathrm{~km}$ de profondeur. tée vers la surface.

La roche fond et produit du magma : un mélange de gaz et de roche fondue.

Tableau 2. Exemple d'un écrit final problématisé concernant le magmatisme en classe de $4^{e}$ (Chalak, 2012 a et b).
Suite à cette étude approfondie des écrits de la séquence ordinaire, nous pouvons formuler des hypothèses concernant la logique de mise en texte de l'enseignante qui apparaît centrée sur la sélection et le tri de la bonne solution parmi les propositions avancées par les élèves (Orange-Ravachol, 2010). Par ailleurs, nous précisons que la consigne de départ : «Propose un dessin très simple d'un volcan en coupe où tu places le lieu d'où viennent les matériaux, avec des hauteurs possibles, avec l'emplacement du sol par rapport à ces matériaux etc. À légender » ne semble pas confronter les élèves à un problème explicatif mais plutôt à un problème descriptif qui invite les élèves à situer le lieu à l'origine du magma. Les écrits initiaux sont des mono-textes propositionnels parce qu'ils donnent à voir les solutions initiales des élèves au problème posé. Le premier écrit intermédiaire produit est un pluri-texte propositionnel qui présente les différentes solutions possibles alors que le deuxième écrit est mono-texte argumentatif qui expose plusieurs arguments dont le but est de participer au tri en favorisant ou défavorisant l'une ou l'autre proposition. Le troisième et le quatrième écrit sont des mono-textes propositionnels qui désignent la bonne solution au problème de l'origine des matériaux volcaniques. Pour mieux comprendre les pratiques de mise en texte de l'enseignante et son mode de fonctionnement qui semble basé sur le tri des solutions au sein d'une situation visiblement peu propice à la problématisation, nous nous proposons d'effectuer une modélisation praxéologique des pratiques ordinaires en jeu dans cette séquence.

\section{Modélisation praxéologique des pratiques enseignantes de mise en texte}

À ce stade, la description de la séquence et l'analyse des écrits élaborés nous permettent d'effectuer une modélisation des pratiques enseignantes en jeu dans la mise en texte du problème en question. Nous nous appuyons sur l'analyse praxéologique de la pratique enseignante telle qu'elle est définie dans la théorie anthropologique du didactique (TAD) (Chevallard, 1997, 1998). Les données dont nous disposons pour cette séquence vont nous permettre d'étudier le bloc pratico-technique (savoir-faire), c'est-à-dire les types de tâches et les techniques et d'accéder à la technologie faisant partie du deuxième bloc technologico-théorique (savoir). Rappelons que 
la mise en texte relève d'un type de tâche enseignant dont l'accomplissement appelle des techniques enseignantes particulières. Pour identifier ces techniques, il s'agit tout d'abord de repérer les tâches effectuées par la classe. Nous avons repéré huit tâches de la classe par ordre chronologique (figure 10) : Produire un dessin simple d'un volcan en coupe où sera place le lieu d'où viennent les matériaux volcaniques; produire une explication commune en groupe et un écrit de groupe; Débattre sur les productions de groupe; Produire un écrit à la fin du débat ; Exploiter collectivement la documentation scientifique ; Produire un écrit suite à l'exploitation de la documentation; Produire un écrit bilan final ; Produire un schéma bilan final et discuter le mécanisme de remontée des matériaux. La réalisation de chacune de ces tâches conduit à l'élaboration d'ostensifs par la classe : oraux et écrits, de natures (initiaux, individuels et finaux) et de formes variées (schémas, textes). Certains ostensifs sont utilisés pour réaliser les tâches suivantes. La modélisation praxéologique des pratiques enseignantes de mise en texte est ainsi obtenue (figure 10):

- En effectuant la tâche qui consiste à produire un dessin simple d'un volcan où le lieu de provenance des matériaux volcaniques est placé, les élèves élaborent individuellement un schéma qu'ils utilisent lors du travail de groupe pour construire des modèles explicatifs communs à chaque groupe. Ces mono-

\section{Tâches de la classe}

Produire un dessin simple d'un volcan en coupe où sera placé le lieu d'où viennent les matériaux volcaniques

Produire une explication commune en groupe et un écrit de groupe

Débattre sur les productions de groupe

Produire un écrit à la fin du débat

Exploiter collectivement la documentation scientifique

Produire un écrit suite à l'exploitation de la documentation

Produire un écrit bilan final

Produire un schéma bilan final et discuter le mécanisme de remontée des matériaux
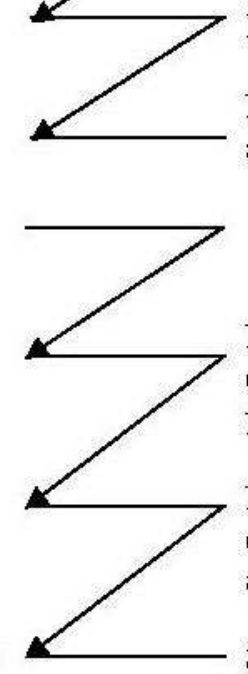

Ostensifs produits-utilisés

Production initiales individuelles (schéma légendé)
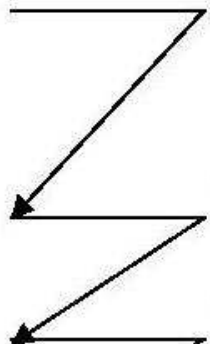

Echanges oraux/arguments sur

les productions de groupe

Ecrit (E1) résumant les

solutions possibles

Echanges oraux/arguments

Ecrit (E2) permettant de valider et d'invalider les solutions déjà proposées

Ecrit bilan final (E3) exposant la solution vraie au problème

Schéma bilan final (E4)

\section{Techniques de l'enseignante}

Identifier-repérer les solutions possibles au problème de l'origine des matériaux volcaniques

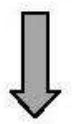

Discuter et critiquer les solutions possibles<smiles>[C]1C=C1</smiles>
Repérer la bonne solution pour le problème de l'origine des matériaux volcaniques

Production d'un ostensif à partir d'une tâche donnée

Ostensif utilisé comme

objet de travail pour la tâche suivante

Figure 10. Modélisation praxéologique des techniques enseignantes de mise en texte du problème de l'origine des matériaux volcaniques. 
textes propositionnels serviront d'appui lors du débat scientifique ce qui conduira à l'élaboration du premier écrit intermédiaire E1 résumant les solutions possibles apparues pour le problème de départ. L'ensemble de cette première phase a pour but, pour l'enseignante, d'identifier et de repérer les solutions possibles envisagées par les élèves au problème de l'origine des matériaux volcaniques. L'écrit El produit à la fin de cette phase est - comme nos analyses précédentes le montrent - un pluri-texte propositionnel qui présente les différentes solutions proposées par les élèves. - La tâche d'exploitation de la documentation scientifique n'utilise pas directement l'écrit E1 mais un document du manuel scolaire (annexe 1). Sa réalisation permet de concevoir un deuxième écrit intermédiaire E2, considéré comme un mono-texte argumentatif, qui valide et invalide les solutions déjà évoquées dans l'écrit El à travers des argumentations. Par conséquent, cette phase vise principalement, pour l'enseignante, la discussion et la critique des solutions possibles avancées lors du débat de classe.

- Lécrit intermédiaire E2 est utilisé pour la production d'un écrit bilan final lui-même servant à la production d'un schéma bilan final. Ces tâches donnent naissance à des écrits finaux qui ne font que présenter la bonne solution au problème de l'origine des matériaux volcaniques posé au départ au sein de monotextes propositionnels (E3 et E4).

Les techniques enseignantes de mise en texte ont permis, suite à la réalisation de tâches par la classe et à la production d'ostensifs, de repérer les solutions possibles que les élèves proposent au problème, puis de critiquer ces solutions afin de retenir une seule solution figurant dans un texte final propositionnel. Du point de vue de la problématisation, par ce cheminement et cette manière de faire, nous pointons un tri de la bonne solution déjà identifié par d'autres recherches (Orange-Ravachol, 2010). Le tri des solutions se fait, au fur et à mesure de l'avancée de la séquence, à travers les différentes tâches réalisées par la classe et les divers niveaux d'écrits successifs produits (mono-textes propositionnels $\rightarrow$ pluri-texte propositionnel $\rightarrow$ mono-texte argumentatif $\rightarrow$ monotexte propositionnel). À partir de cette modélisation praxéologique et de l'analyse précédente des écrits, nous pouvons avancer que les technologies justi- fiant ces techniques de mise en texte se basent sur les conceptions épistémologiques de l'enseignante qui considère les savoirs scientifiques comme des solutions vraies et factuelles aux problèmes posés. Par ailleurs, nous signalons que les techniques enseignantes de mise en texte basées sur le repérage et le tri des solutions ainsi que les technologies qui les justifient ne sont pas spécifiques aux sciences de la Terre. Elles peuvent très bien permettre d'accomplir la tâche enseignante de mise en texte d'un problème en sciences de la vie ou en sciences physiques par exemple. Cependant, certaines tâches réalisées par la classe, comme "produire un dessin simple d'un volcan », sont spécifiques aux sciences de la Terre par leur objet tandis que d'autres comme « débattre sur les productions de groupe » peuvent être considérées comme étant des tâches génériques.

Cette première analyse, qui porte sur les textes produits au cours de la séquence et les pratiques de mise en texte, nous a permis de décrire les techniques enseignantes pour une activité de mise en texte qui visiblement ne garde pas de traces de la problématisation. Cependant, nous ne pouvons pas nous arrêter au niveau de cette analyse car les savoirs ne peuvent se réduire aux seuls textes écrits. D'où la nécessité de se pencher sur les moments de travail de la classe ayant conduit à la production de ces textes : Comment ces textes ont-ils été construits en situation ? Comment s'est déroulée leur mise en texte? Retrouve-t-on les traces de la problématisation et de la construction des raisons lors de la mise en texte orale ? C'est ainsi que nous partons à la recherche des pratiques ayant abouti à la construction des écrits lors de deux phases de travail de notre séquence : le débat collectif et l'exploitation de la documentation scientifique. La première phase a permis le passage des écrits explicatifs de groupe à l'écrit intermédiaire El et la deuxième de l'E1 à l'E2.

\section{Au cours du débat : une focalisation sur les solutions et leur repérage}

L'écrit intermédiaire El correspond à une liste de trois propositions différentes au problème de l'origine des matériaux volcaniques : la lave existe au niveau du noyau, soit dans une poche pas très profonde ou dans le volcan lui-même. Nous nous intéressons à l'étude de l'activité de mise en texte de l'enseignante en interaction avec les élèves pendant 
la situation de débat. Cette dernière a été pensée dans le but de mettre en jeu les conceptions des élèves sur l'origine du volcanisme. Pour suivre les pratiques enseignantes de mise en texte au cours de cette phase, nous avons construit la macrostructure de ce débat (figure 11) qui représente un résumé global du débat avec des propositions de type question/réponse et qui donne à voir la dynamique de cette phase (Fabre

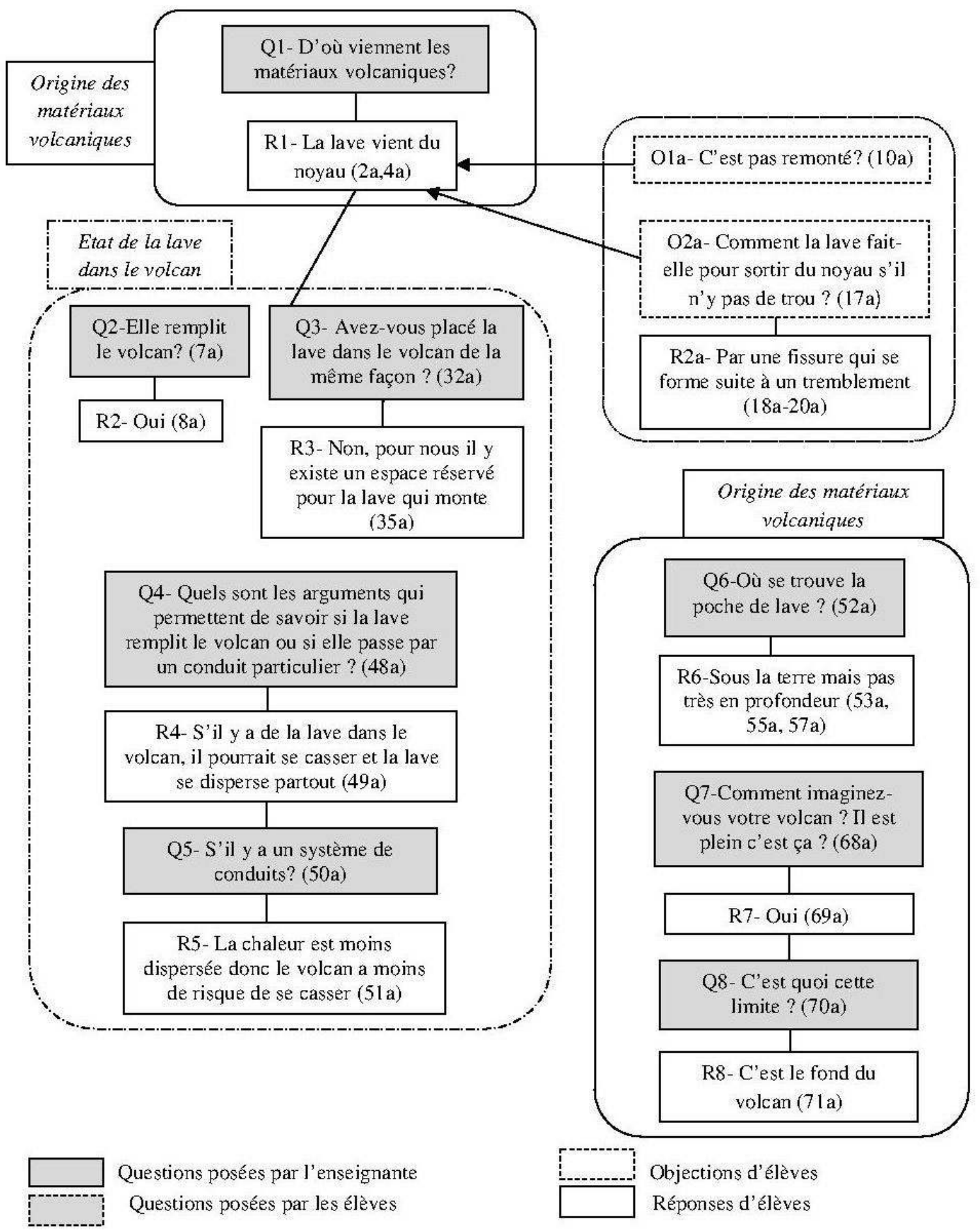

Figure 11. Macrostructure du débat scientifique de la classe de $4^{e}$ concernant l'origine des matériaux volcaniques. 
\& Orange, 1997 ; Fabre, 1999). Nous avons indiqué les questions traitées $(\mathrm{Q})$, les réponses évoquées $(\mathrm{R})$ et les objections formulées (O). Ensuite, nous avons regroupé les propositions qui traitent du même problème. Les traits expriment les liens entre les différentes propositions et les chiffres correspondent aux numéros des interventions dans la transcription du débat. Certaines propositions ont été reformulées ou simplifiées tout en prenant le soin de garder leur sens initial dans le débat.

Cette macrostructure nous permet de faire plusieurs remarques :

- Trois problèmes ont été travaillés pendant le débat scientifique : celui de l'origine des matériaux volcaniques, de l'état de la lave dans le volcan (remplissage ou non du volcan par la lave) et de la remontée des matériaux. Les deux premiers ont été introduits par l'enseignante alors que le troisième l'a été par les élèves prenant appui sur le schéma et la présentation du premier groupe. Nous remarquons aussi que le problème de l'origine des matériaux volcaniques est traité au début et à la fin du débat.

- Les questions sont souvent posées par l'enseignante. Elles demandent de simples explicitations (Q2, Q3, Q4, Q6, Q7, Q8) ou des argumentations (Q4) qui donnent lieu à une seule réponse (R2-R8). Les élèves interviennent à travers deux objections (O1a, O2a) qui remettent en cause certaines informations qui figurent sur le schéma du premier groupe. Ainsi, cette situation porte des particularités qui l'éloignent des véritables caractéristiques des débats scientifiques qui sont des moments privilégiés d'échanges entre les élèves. Mais nous continuerons à parler de débat, dans ce qui suit, afin de préserver la continuité dans notre exposé.

Pour étudier ce qui se joue au cours de la phase de débat du point de vue des savoirs, de la problématisation et des pratiques de mise en texte, cette macrostructure que nous venons de décrire ne suffit pas à elle seule. Il est alors nécessaire de reprendre en détail la transcription des échanges et un certain nombre d'interventions et d'interactions d'enseignants et d'élèves. Nous rappelons que deux problèmes ont été traités en plus du problème principal de l'origine des matériaux volcaniques : le problème de remontée des matériaux volcaniques et celui du remplissage ou non du volcan par la lave. Regardons de plus près, comment le groupe classe introduit ces problèmes, les traite et passe de l'un à l'autre.

\section{La remontée des matériaux volcaniques}

Prenons le détail des interventions qui ont introduit ce problème suite à la présentation du schéma du premier groupe (figure 9) qui évoque une lave arrivant au volcan du noyau terrestre:

\begin{tabular}{|c|c|}
\hline $10 \mathrm{a}$ & Alexandre. Euh c'est c'est pas remonté ? \\
\hline $11 \mathrm{a}$ & P. Demande à Amandine. \\
\hline $12 \mathrm{a}$ & Amandine. Comment ça? \\
\hline $13 a$ & Alexandre. Le trait au milieu, c'est la sortie? \\
\hline $14 \mathrm{a}$ & Amandine. C'est la surface du sol. \\
\hline $15 \mathrm{a}$ & Alexandre. Ah oui, c'est donc, c'est un dessin? \\
\hline $16 \mathrm{a}$ & Amandine. Hum. \\
\hline $17 \mathrm{a}$ & $\begin{array}{l}\text { Franck. Comment la lave qui sort du noyau déjà je } \\
\text { comprends pas, comment elle fait pour passer dans } \\
\text { le volcan s'il n'y a pas de trou pour qu'elle passe? } \\
\text { parce ce que là le sol, il y a... }\end{array}$ \\
\hline $18 \mathrm{a}$ & Emmanuel. Il y a la fissure. \\
\hline $19 \mathrm{a}$ & Franck. Ah ben non, je ne vois pas de fissure. \\
\hline $20 \mathrm{a}$ & $\begin{array}{l}\text { P. Chut Chut. Emmanuel! Emmanuel peut propo- } \\
\text { ser une solution à Amandine après elle pourra euh } \\
\text { alors vas-y. }\end{array}$ \\
\hline $21 \mathrm{a}$ & $\begin{array}{l}\text { Emmanuel. Ben quand il y a le tremblement, quand } \\
\text { la fissure elle se forme et puis euh la lave elle monte. }\end{array}$ \\
\hline $22 \mathrm{a}$ & Franck. Non mais là... \\
\hline $23 a$ & $\begin{array}{l}\text { P. Donc tais-toi pour l'instant, la fissure où ? est-ce } \\
\text { que tu pourrais aller au tableau et essayer d'expli- } \\
\text { quer avec le dessin d'Amandine où est-ce que tu } \\
\text { mettrais ta fissure? Vas-y, tu restes là quand même } \\
\text { Amandine. Après, tu laisses Emmanuel parler après } \\
\text { je t'écoute. }\end{array}$ \\
\hline $24 \mathrm{a}$ & Franck. J'ai même pas fini ma question! \\
\hline $25 a$ & $\begin{array}{l}\text { Emmanuel. Ben là, enfin... (Emmanuel montre au } \\
\text { niveau de la surface du sol sous le volcan). }\end{array}$ \\
\hline $26 a$ & $\begin{array}{l}\text { P. D'accord. Donc il y aurait une fissure qui permet } \\
\text { de laisser passer. Jason? }\end{array}$ \\
\hline
\end{tabular}

Le modèle présenté par un membre du premier groupe attribue l'origine de la lave au noyau de la Terre et se rapporte ainsi à une explication centrale situant cette origine en référence à la Terre. Lorsque Alexandre (10a) et Franck (17a) et s'interrogent sur la remontée du magma et avancent deux objections en référence à l'affiche du premier groupe, les échanges s'arrêtent à partir du moment où Emmanuel (18a, 21a) évoque une fissure provoquée par un tremblement de terre qui autoriserait la remontée du magma. L'objection de Franck, sous forme d'une question, prend en compte les informations du schéma et remet en cause la possibilité 
d'une remontée de la lave du noyau vers le volcan sans qu'il y ait un «trou » facilitant ce passage. Ce n'est pas tant l'origine de la lave telle que proposée au niveau du noyau qu'il conteste mais la représentation schématique qui ne suggère pas son évacuation du noyau et qui la place pourtant au niveau du volcan. Cette remontée semble nécessaire du point de vue des élèves pour le fonctionnement du volcan et pour que la proposition du premier groupe puisse fonctionner. Derrière ces interventions figure un lien qu'effectuent les élèves entre l'origine des matériaux et leur arrivée à la surface et la nécessité d'un système (ou d' « un trou ») qui puisse permettre au magma de passer du noyau vers le volcan. C'est le problème de la remontée du magma qui a préoccupé les élèves et non pas celui de sa formation puisqu'il préexiste à l'endroit mentionné par le premier groupe, au niveau du noyau. La proposition d'Emmanuel (18a, 21a) sous-entend un lien de causalité simple entre les séismes et le déclenchement des éruptions qui attire assez souvent la focalisation des élèves. Ceci est lié à un raisonnement linéaire simple : tremblement $\rightarrow$ fissure $\rightarrow$ montée de la lave. Suite à la demande de l'enseignante, Emmanuel localise sa fissure sur le schéma au niveau de la surface du sol sous le volcan ce qui semble susciter l'accord de l'enseignante qui conclut sur ce problème et met fin à la discussion par les interventions suivantes : «26a. P. D'accord. Donc il y aurait une fissure qui permet de laisser passer. [...]» et plus loin «32a. P. Oui, alors, est-ce que vous aviez réfléchi à ça ? Une fissure éventuelle qui pourrait emmener euh la matière depuis le noyau jusqu'à la surface ? Bon maintenant que ça c'est un petit peu euh évoqué $[\ldots]$ ». Mais nous nous demandons pourquoi l'enseignante n'est-elle pas allée plus loin avec les élèves en leur posant des questions qui auraient pu les engager dans une activité de réflexion et de problématisation plus poussée : Quelles sont les raisons qui fondent l'idée d'Emmanuel et qui font que la fissure se forme là où il l'a placée et pas ailleurs? Comment ferait le magma pour passer du noyau et être évacué au niveau de la fissure placée au niveau de la surface du sol ? Concernant l'origine de la lave, quelles sont les raisons derrière l'idée d'une origine située au niveau du noyau ? Pourquoi la lave viendrait-elle du noyau terrestre et pas d'un autre endroit?

Du point de vue de la problématisation, dès la première phase du débat, les interventions des élèves portent les germes d'une nécessité fonctionnelle: celle de la remontée du magma. Mais les échanges s'arrêtent à partir du moment où l'un des élèves évoque une fissure provoquée par un tremblement de terre qui autoriserait la remontée du magma. Quant aux interventions enseignantes, nous remarquons qu'elles ne poussent pas les élèves au-delà des solutions éventuelles introduites pour repérer les raisons qui sous-tendent ces solutions. Ainsi, l'écrit El ne garde de ces moments d'échanges que la solution proposée par le premier groupe : « la lave vient du noyau de la terre $»$.

\section{Le remplissage du volcan par la lave}

C'est l'enseignante qui introduit, à deux reprises, le problème du remplissage ou non du volcan par la lave qui semble la préoccuper. D'abord, par une question adressée à Amandine $(7 \mathrm{a})^{7}$ lors de la présentation des idées du premier groupe en référence aux données de l'affiche:

\begin{tabular}{|l|l|}
\hline 7a & $\begin{array}{l}\text { P. Au milieu de la terre, au centre de la terre et donc } \\
\text { ensuite la lave remonte dans le volcan et euh donc } \\
\text { euh là ici vous l'avez mise, elle remplit le volcan c'est } \\
\text { ça? }\end{array}$ \\
\hline $8 \mathrm{a}$ & Amandine. Hum. \\
\hline
\end{tabular}

Puis, elle y revient au cours du débat - après l'introduction du problème de la remontée du magma à travers l'intervention $32 \mathrm{a}$ qui ouvre la voie à la discussion de ce problème. La classe est ainsi orientée vers un autre problème en rapport avec le schéma du premier groupe mais cette fois-ci pointé par l'enseignante.

\begin{tabular}{|l|l|}
\hline $32 a$ & P : Oui, alors, est-ce que vous aviez réfléchi à ça ? \\
& Une fissure éventuelle qui pourrait emmener euh \\
la matière depuis le noyau jusqu'à la surface ? Bon \\
maintenant que ça c'est un petit peu euh évoqué, est- \\
ce que vous avez tous représenté le volcan comme \\
l'ont fait euh, les élèves du groupe d'Amandine? \\
Vous avez placé la lave de la même façon?
\end{tabular}

Corentin (35a) évoque la présence d'un « espace réservé », dans le schéma de son groupe, pour la lave dans le volcan. Suite à l'explication de Thomas (45a) qui précise que la lave monte à travers l'espace qui lui est réservé lorsque le volcan est en éruption, l'enseignante effectue une comparaison entre le schéma du premier groupe (figure 9) et celui du deuxième groupe (figure 8). Puis, elle demande aux 
élèves d'apporter des argumentations qui permettraient d'appuyer l'un ou l'autre modèle : « 48a. P. [...] Est-ce qu'on a des arguments déjà pour dire le volcan est plein de lave ou bien il y a des passages, des conduits pour la lave? À votre avis, quels sont les arguments qui pourraient dire, j'ai une marmite ici pleine de lave ou bien j'ai un conduit plein de lave dans le volcan, comment est-ce qu'on pourrait argumenter ça ? "Seule, cette intervention demande aux élèves d'argumenter dans le but de trancher entre deux idées. Corentin (49a-5la) indique alors que si la lave remplissait le volcan, ce dernier pourrait s'effondrer, idée que l'enseignante valorise en la reprenant et la reformulant. Alors qu'avec le système de conduit de son groupe, le volcan court moins ce risque car la chaleur de la lave sera moins dispersée.

\begin{tabular}{|c|c|}
\hline $35 a$ & $\begin{array}{l}\text { Corentin. Nous on a fait ben comme euh, une montée } \\
\text { de lave quoi, on a fait euh un espace réservé pour la } \\
\text { lave qui monte. }\end{array}$ \\
\hline $36 a$ & P. Dans le volcan? \\
\hline $37 \mathrm{a}$ & Corentin. Oui. \\
\hline $38 \mathrm{a}$ & $\begin{array}{l}\text { P. D'accord, c'est-à-dire, vous vous avez proposé, } \\
\text { dans le volcan, si j'arrive à le mettre en même temps, } \\
\text { un espace ici, je sais pas si ça va tenir, un petit } \\
\text { peu compliqué pour le recaler. Voilà le dessin que } \\
\text { proposait Corentin, enfin le groupe de Corentin, pas } \\
\text { Corentin tout seul bien sûr. Donc avec l'idée, c'est ça } \\
\text { que tu expliquais... Thomas vas-y donc montre ce } \\
\text { qui est expliqué. }\end{array}$ \\
\hline $45 a$ & $\begin{array}{l}\text { Thomas. Ben on le voit pas très bien mais là en fait } \\
\text { euh c'est comme si c'était une grosse fuite de lave. }\end{array}$ \\
\hline $46 a$ & P. Oui. \\
\hline $47 \mathrm{a}$ & $\begin{array}{l}\text { Thomas. Donc euh là on va suivre la lave qui était } \\
\text { accroché avec. Ben quand il est pas en éruption ben } \\
\text { la lave elle monte pas et quand il est en éruption eh } \\
\text { ben elle monte. }\end{array}$ \\
\hline $48 \mathrm{a}$ & $\begin{array}{l}\text { P. D'accord. Donc la différence entre le schéma } \\
\text { d'Amandine et le vôtre, d'Amandine de Félix et } \\
\text { d'Ivana, c'est que là la lave elle est dans un conduit } \\
\text { particulier comme tu l'as évoqué Corentin, alors } \\
\text { qu'elle remplit complètement le volcan chez les } \\
\text { autres. Qu'est-ce que vous en pensez les autres } \\
\text { par rapport aux schémas de ces deux modèles ? } \\
\text { On voit déjà, vous pouvez retourner tous les deux } \\
\text { vous asseoir on va discuter là-dessus. Alors qu'est- } \\
\text { ce qu'on peut, qu'est-ce que vous en pensez ? Est-ce } \\
\text { qu'on a des arguments déjà pour dire le volcan est } \\
\text { plein de lave ou bien il y a des passages, des conduits } \\
\text { pour la lave? À votre avis, quels sont les arguments } \\
\text { qui pourraient dire, j'ai une marmite ici pleine de } \\
\text { lave ou bien j'ai un conduit plein de lave dans le } \\
\text { volcan, comment est-ce qu'on pourrait argumenter } \\
\text { ça? Corentin? }\end{array}$ \\
\hline $49 a$ & $\begin{array}{l}\text { Corentin. Ben s'il y a de la lave dans le volcan euh, il } \\
\text { pourrait euh se casser quoi et la lave elle se disperse } \\
\text { partout. }\end{array}$ \\
\hline
\end{tabular}

\begin{tabular}{|l|l|}
\hline 50a & $\begin{array}{l}\text { P. Bon. L'idée c'est que si j'ai vraiment un volcan qui } \\
\text { est rempli de lave comme ça, tu dis eh bien les bords } \\
\text { du volcan pourraient se casser et à ce moment-là, } \\
\text { ben le volcan lui-même pourrait s'ouvrir de partout } \\
\text { et la lave pourrait sortir partout. D'accord. Si on } \\
\text { est ici avec un volcan avec un système de conduits, } \\
\text { Corentin? }\end{array}$ \\
\hline $51 \mathrm{a}$ & $\begin{array}{l}\text { Corentin. Ben la chaleur est moins dispersée, donc } \\
\text { euh, le volcan lui-même à moins de risque de se } \\
\text { casser. }\end{array}$ \\
\hline
\end{tabular}

Par la suite, l'enseignante fait le tour des schémas des autres groupes pour faire le point et les comparer du point de vue de leur représentation de la lave au niveau du volcan (52a). Puis, elle revient sur le problème de l'origine des matériaux volcaniques (52a).

\begin{tabular}{|l|l|}
\hline $52 a$ & $\begin{array}{l}\text { P. D'accord. Est-ce qu'il y a d'autres euh proposi- } \\
\text { tions, par rapport à ce que vous avez mis vous hein. } \\
\text { Le groupe de, le groupe de Simon a aussi proposé } \\
\text { un, il me semble un passage avec un conduit, le } \\
\text { groupe par contre de Jessica comme le groupe de } \\
\text { euh qui est-ce, de Raphaël, vous avez proposé un } \\
\text { peu plus ce modèle là avec un volcan qui est rempli } \\
\text { de euh, d'éléments bouillonnants ou de lave. C'est } \\
\text { ça? Vous vous y retrouvez dans un des deux sché- } \\
\text { mas tout le monde? Bon, eh bien on va déjà on va } \\
\text { garder l'argument éventuellement de la discussion, } \\
\text { de savoir est-ce que le volcan est rempli de lave } \\
\text { ou bien est-ce que la lave passe par des endroits } \\
\text { précis. Maintenant on continue, le groupe premier } \\
\text { groupe pour lequel on travaille, nous propose que } \\
\text { l'origine de la lave, elle vient du centre de la terre, le } \\
\text { deuxième groupe, il se trouve où cet ensemble, cette } \\
\text { espèce de poche que vous avez représenté, Corentin, } \\
\text { Thomas et puis euh Kevin? }\end{array}$ \\
\hline
\end{tabular}

Nous pouvons ainsi constater que le problème du remplissage du volcan, en lien avec l'affiche du premier groupe, préoccupe l'enseignante. Elle l'introduit à deux reprises et demande des argumentations pour trier les deux modèles opposés : un volcan plein de lave vs présence d'un conduit qui permet l'ascension de la lave dans le volcan. L'enseignante semble chercher des alternatives d'idées ou de solutions pour préparer son écrit intermédiaire qu'elle prévoit être sous forme de solutions à départager (écrit E1). Les argumentations avancées par les élèves ne figurent pas dans le texte intermédiaire El mais la proposition qui postule que la lave serait dans le volcan fermé à la base est inspirée du modèle du groupe de Corentin (figure 8). 


\section{Le retour à l'origine des matériaux volcaniques à la fin du débat}

La fin du débat, qui marque le retour au problème de l'origine des matériaux volcaniques, se caractérise par une phase où une série de questions est posée par l'enseignante. Ces derniers ont pour but d'aider les élèves à mieux expliciter leurs idées concernant l'origine du magma qui figure sur leur production de groupe. Ces questions demandent de simples explicitations et c'est l'enseignante qui prend en charge la sélection des schémas en adressant directement les questions aux membres du groupe concerné. Les interventions ci-dessous illustrent les propos que nous venons d'avancer. L'enseignante semble poser des questions pour clarifier les idées des élèves de façon à repérer les solutions et préparer le tri qui suivra.

\begin{tabular}{|l|l|}
\hline $52 \mathrm{a}$ & $\begin{array}{l}\text { P. [...] le deuxième groupe, il se trouve où cet } \\
\text { ensemble, cette espèce de poche que vous avez } \\
\text { représentée, Corentin, Thomas et puis euh Kevin? }\end{array}$ \\
\hline $53 \mathrm{a}$ & Thomas. Ben il est en dessous de la terre. \\
\hline $54 \mathrm{a}$ & $\begin{array}{l}\text { P. En dessous de la terre, est ce qu'il est euh aussi } \\
\text { profond que la représentation d'Amandine? }\end{array}$ \\
\hline $55 \mathrm{a}$ & Thomas. Ben, pas trop. \\
\hline $56 \mathrm{a}$ & P. Pas trop, ça c'est pas une réponse. \\
\hline $57 \mathrm{a}$ & \begin{tabular}{l} 
Thomas. Pas trop loin mais euh. \\
\hline $58 \mathrm{a}$
\end{tabular} $\begin{array}{l}\text { P. D'accord. Il est moins profond, il est quand même } \\
\text { en profondeur, moins profond. Qu'est-ce que vous } \\
\text { avez pensé, vous les autres groupes? Sur l'origine } \\
\text { de la lave. J'ai un dessin comme ça par exemple. } \\
\text { Vous allez me dire d'où ça vient ? Je retiens pour } \\
\text { l'instant ceux-là, celui-là, le groupe du fond, Jessica, } \\
\text { mais c'est pas Jessica qui répond forcément. Où ça } \\
\text { va, ça ? Allez-y hein parce que, racontez-nous votre } \\
\text { idée. Alors qui est-ce qui parle, Clodevi ? }\end{array}$ \\
\hline
\end{tabular}

Le débat a permis la réalisation d'un état des possibles ce qui renvoie à un aspect de la problématisation mais les argumentations autour de l'impossibilité des possibles ne sont pas retenues. Cette phase d'échange se place plus au niveau des solutions du problème qu'au niveau des raisons. L'écrit intermédiaire El élaboré à la fin, recopié par les élèves et qualifié de pluri-texte propositionnel ne garde que les solutions proposées au problème. Il ne reprend rien de certains arguments et problèmes ayant émergés lors du débat. Cette manière de faire se comprend si, comme nous l'admettons, la pratique de l'enseignante est basée sur l'identification des idées des élèves et leur tri par l'étude de documents ou d'expériences.
Ses intentions semblent orientées prioritairement vers l'identification des solutions possibles et non pas vers un travail de problématisation et de construction des nécessités dans une situation qui apparaît peu propice dès le départ à ce travail. Le débat, prépare la mise à l'épreuve des modèles proposés à travers la documentation scientifique qui permettra de choisir entre les propositions de solutions celle qui est vrai : «68a. P. [...] bon alors les questions qui vont arriver, ça va être est-ce que la lave vient du noyau, est-ce qu'elle vient d'une poche qui est pas trop profonde, ou bien point d'interrogation on revient hein [...]». À côté d'un souci de donner la parole aux élèves, l'enseignante contrôle le débat et joue, comme l'étude de la transcription des échanges le montre, un rôle dans l'orientation vers certains problèmes dans le débat. Nous avons peu de traces de problématisation dans les échanges entre l'enseignante et les élèves. Ainsi, nous notons une difficulté à problématiser et à mener une mise en texte orale de nature problématisée dans la classe. De plus, peu de temps a été consacré à ce débat et les cinq groupes ne sont pas passés exposer et débattre leur modèle comme le préconise la méthodologie du débat (Orange, 2003). On se limite à la présentation du modèle d'un premier groupe et les autres sont présentés rapidement en comparaison avec celui-ci et sous le contrôle de l'enseignante.

Le travail se poursuit avec l'étude des documents où les solutions proposées par les élèves sont discutées.

\section{Au cours de la confrontation avec la documentation scientifique : critique des solutions possibles et leur tri}

La phase d'exploitation collective du document scientifique qui montre l'enregistrement de l'activité sismique sous un volcan (annexe 1) conduit à l'écrit intermédiaire E2. Celui-ci décrit le document, emmène une explication argumentée à l'état liquide de la zone sans séismes observée sous le volcan et avance des argumentations en faveur de l'impossibilité d'une lave provenant du centre de la Terre puisque ce dernier est solide, contrairement à ce que les élèves peuvent penser.

Dès le début de la séance (séance 3), l'enseignante oriente les élèves vers une problématique de choix, de tri des propositions déjà repérées lors du débat afin de retenir celle répondant réellement au 
problème posé : « 2b. P. [...] Alors maintenant eh bien pour essayer de savoir, si euh y a une idée dans ces trois groupes qui devait émerger comme étant réellement l'origine de la matière, il faudrait donc qu'on puisse vérifier, est-ce qu'on a un moyen de vérifier où se trouve le, la lave en fait par rapport au volcan. Quel est l'état de la lave par rapport au volcan et par rapport à la Terre? »

Nous commençons cette analyse par un incident que nous relevons et qui marque le début de cette phase d'exploitation de la documentation scientifique.

\section{Émergence de la relation de causalité simple entre l'activité sismique et volcanique}

L'étude de la transcription des échanges montre que les élèves, au début de l'exploitation collective du document scientifique, évoquent une relation de causalité simple entre les séismes et le volcanisme non attendue par l'enseignante : «24b. Alexandre. L'activité sismique euh, elle entraîne l'activité volcanique ». Cette thèse a été soutenue et argumentée par Alexandre «26b. Ben quand le sol il tremble, ça pourrait faire euh, ça entraînerait des euh, des bouillonnements. » suivi d'Emmanuel « 32b. Oui, parce que quand la terre tremble ça peut faire une rupture euh dans le volcan et euh la lave euh » et de Maxime «36b. Ben, ça peut oui, ça peut faire une rupture et ça peut monter la lave ». L'émergence de ce problème pousse l'enseignante à solliciter une justification des propositions, puis à demander aux élèves de rappeler la définition et l'origine d'un foyer sismique, enfin à attirer l'attention des élèves sur le fait que les foyers sismiques ne sont pas enregistrés dans le volcan mais bien en-dessous. L'enseignante essaye de régler ce dilemme mais, face aux argumentations des élèves, de plus en plus accrochés à cette idée, elle poursuit et reformule la question mais cette fois-ci en inversant le lien de causalité évoqué par les élèves afin de les déstabiliser « 4lb. P. [...] est-ce que ce sont les séismes qui vont entraîner le volcanisme ou est-ce que c'est le volcanisme qui va entraîner les séismes ? ». Alexandre n'exclut pas alors la possibilité que ce soit le volcanisme qui puisse entraîner les séismes, les pierres rejetées du volcan pouvant créer des séismes. Après les multiples tentatives pour régler le problème, l'enseignante ferme la situation et décide de mettre le problème de côté pour y revenir plus tard.

Mais nous remarquons que cette situation perturbe l'enseignante et la met dans un embarras. Elle rappelle constamment au cours de la séance que le problème de la relation entre l'activité volcanique et sismique sera traité ultérieurement. Pour elle, le recours au document doit permettre de trancher entre les solutions possibles déjà identifiées. Ce document convoque les séismes comme méthode d'étude des volcans (annexe 1) mais les élèves partent sur une relation de causalité entre les deux phénomènes. Ainsi, lorsqu'elle demande au début de la phase d'exploitation « 23b P. [...] Quel lien peut-il y avoir entre les foyers des séismes et une activité volcanique ? [...]», l'enseignante s'attend à ce que les élèves s'appuient sur les données sismiques du document pour localiser la poche de magma. Mais la focalisation des élèves sur des éléments de leurs conceptions détourne les apports du document et sa participation dans le tri des solutions.

Nous pointons ici la difficulté de l'enseignante à soustraire les élèves de la causalité simple prédominant dans leurs modes de raisonnements dans une classe qui ne semble pas travailler sur la tâche qu'elle a prévue. Ce problème a émergé contre l'attente de l'enseignante qui a essayé de le régler à plusieurs reprises pour finir par le suspendre. Par la suite, l'étude du document scientifique reprend et nous verrons, dans ce qui suit, comment l'écrit intermédiaire E2 a été construit.

\section{Construction de l'écrit intermédiaire E2}

L'enseignante pousse les élèves, dans un premier temps, à décrire le document montrant la position des foyers sismiques situés sous le volcan, puis reformule leurs idées pour finir par un texte qui décrit le document « on observe des séismes alignés sous le volcan mais en deux groupes séparés par une zone sans séismes ». Ensuite, l'explication de la zone sans séismes présente sous le volcan et qui correspond à une zone liquide a été introduite par l'enseignante (65b) après la lecture du texte qui accompagne le document par Lou Anne. Ceci a permis de valider la deuxième proposition de l'écrit El précédent (65b-66b) : 


\begin{tabular}{|l|l|}
\hline $65 \mathrm{~b}$ & $\begin{array}{l}\text { 65b. P. Alors qu'est-ce que l'on vous dit à ce niveau- } \\
\text { là dans cette explication. On vous dit, lorsqu'il y a } \\
\text { séismes les ondes sismiques ne se propagent jamais } \\
\text { dans les liquides. D'autre part, pour qu'il puisse y } \\
\text { avoir rupture, il faut qu'on soit dans du matériau } \\
\text { solide. D'accord. Donc on suggère que la zone qui } \\
\text { se trouve entre les deux répartitions de foyers } \\
\text { sismiques, est une zone où il ne peut pas y avoir de } \\
\text { rupture. D'accord, si cette zone on peut pas avoir de } \\
\text { ruptures, c'est que probablement on est dans une } \\
\text { zone liquide. D'accord. Par rapport aux propositions } \\
\text { qu'on a faites, on est quand même sous un volcan } \\
\text { dans une activité volcanique. Eh bien on va dire, } \\
\text { cette zone où il n'y a pas de possibilités de rupture, } \\
\text { cette zone liquide est une zone où est stocké le } \\
\text { magma. D'accord. À quoi ça ressemble dans les } \\
\text { propositions que vous aviez faites. Est-ce que c'est } \\
\text { la première proposition qui disait eh bien euh la } \\
\text { lave vient du magma, non, du centre du noyau de } \\
\text { la terre, est-ce que c'est la lave est dans une poche } \\
\text { en profondeur, est-ce que c'est la lave est dans le } \\
\text { volcan bien fermé, rempli. Euh, Corentin? }\end{array}$ \\
\hline $66 \mathrm{~b}$ & Corentin : La lave est dans une poche. \\
\hline
\end{tabular}

Devant les efforts faits pour déconnecter les phénomènes volcaniques et sismiques et pour faire des séismes un moyen d'étude du volcan, l'enseignante prend en charge l'explication de la zone sans séismes observée sous le volcan. L'écrit intermédiaire E2 reprend ce que ce l'enseignante venait d'évoquer : «Cette zone est une zone liquide (où il n'y a pas de ruptures, donc pas de foyers sismiques). Elle correspond, probablement, à un réservoir de magma. »

Après avoir validé la bonne solution au problème posé, il s'agit d'infirmer les deux propositions restantes de l'écrit El. Le document scientifique (annexe 1) apporte uniquement des informations qui permettent de valider la bonne solution. Les deux autres propositions ont été réfutées oralement. D'abord, l'enseignante demande aux élèves de rappeler les arguments qu'ils avaient déjà évoqués pendant le débat par rapport à la possibilité d'avoir un volcan rempli ou non de lave. Ces arguments, qui évoquent l'effondrement du volcan s'il était rempli de lave, avaient déjà été mentionnés par Corentin et leur rappel a permis d'infirmer la proposition : « la lave est dans le volcan qui est fermé à la base ». Cependant, nous ne retrouvons pas leur trace dans le texte écrit E2 à la fin de cette phase ni auparavant dans l'écrit El. Ensuite, la troisième proposition de l'écrit El qui évoque une lave provenant du noyau pour alimenter le volcan a été rejetée par l'enseignante (81b). En effet, l'augmentation de la température avec la profondeur laisse penser que le noyau est liquide mais la pression qui augmente en même temps rend celui-ci solide. Ces argumentations ont été évoquées dans l'écrit E2.

\begin{tabular}{|l|l|}
\hline $81 b$ & $\begin{array}{l}\text { P. [...] Maintenant est-ce que il y a un lien avec le } \\
\text { noyau de la terre comme le reprend ce dessin-là. Il } \\
\text { y avait un petit peu plus sur leurs dessins person- } \\
\text { nels hein qui était l'idée qu'il y avait un lien entre } \\
\text { le noyau et le remplissage de lave. Il faudrait donc } \\
\text { savoir si le noyau de la terre il est parfaitement, se, } \\
\text { liquide. D'accord. Est-ce qu'il contient une grosse } \\
\text { masse de magma et finalement, il alimente les réser- } \\
\text { voirs, de tous les volcans du monde. Alors comment } \\
\text { le savoir ? Là c'est plus difficile, on peut aussi se } \\
\text { baser sur le passage des ondes sismiques, pour aller } \\
\text { vérifier comment est le noyau mais on peut aussi se } \\
\text { baser sur des données physiques. Quand on va à l'in- } \\
\text { térieur de la terre, plus on s'enfonce dans la terre } \\
\text { plus la température augmente. Et la température au } \\
\text { niveau du noyé, noyau pardon elle est extrêmement } \\
\text { importante, par rapport au fait de rendre liquide } \\
\text { quelque chose, c'est intéressant d'avoir une tempéra- } \\
\text { ture importante. On l'a dit tout à l'heure que c'était la } \\
\text { chaleur qui faisait fondre. Vous vous rappelez de ça? } \\
\text { Le problème est que plus on s'enfonce dans la terre, } \\
\text { plus il y a un autre facteur qui augmente et qui est } \\
\text { la pression. Et quand on arrive au cour de la terre, } \\
\text { la pression elle est colossale et du coup, la pression } \\
\text { est tellement forte au centre de la terre qu'elle va } \\
\text { rassembler les molécules, et empêcher qu'elles ne } \\
\text { forment un liquide. Et le noyau de la terre, pour } \\
\text { le groupe d'Amandine, il est parfaitement solide. } \\
\text { D'accord. Donc il ne peut pas alimenter en magma } \\
\text { les réservoirs magmatiques. D'accord. [...]. }\end{array}$ \\
\hline
\end{tabular}

L'enseignante termine cette partie en précisant aux élèves le lieu d'où vient le magma. Il viendrait de zones situées jusqu'à $200 \mathrm{~km}$ de profondeur où la roche a la possibilité de fondre pour remplir les réservoirs de magmas situés aux alentours de $70 \mathrm{~km}$. Cette précision n'a pas été prise en compte dans le texte E3 ni dans le schéma bilan E4 où simplement « une origine profonde » est évoquée.

La phase de documentation scientifique est marquée par une logique de tri du bon modèle à partir des documents et des arguments sous une importante présence de l'enseignante comme le montre l'analyse effectuée. Ce fonctionnement ordinaire de mise en texte diffère d'un fonctionnement qui privilégierait la problématisation où la recherche des conditions de possibilités des solutions est primordiale. Ce cadre théorique a pour but de faire passer les élèves de leurs idées spontanées aux raisons et non pas des solutions possibles à la bonne solution comme c'est visiblement le cas dans cette séquence ordinaire. De plus, la logique de tri repérée présente des difficultés pour la classe car les documents font appel, semble-t-il, à des arguments difficiles pour les élèves. La bonne solu- 
tion est d'abord identifiée puis les autres infirmées. L'écrit E2 reprend les argumentations évoquées lors de ce moment mais ce sont celles de l'enseignante. Cependant, les arguments qui infirment la deuxième proposition de l'écrit El et qui ont été évoqués pendant cette phase n'ont pas été repris par l'E2.

\section{DIFFICULTÉS D'UNE MISE EN TEXTE PROBLÉMATISÉ DANS LE DOMAINE DU MAGMATISME}

L'étude de la séquence ordinaire de $4^{\mathrm{e}}$ permet de montrer que la pratique de mise en texte enseignante est dominée par une logique de repérage puis de tri des solutions possibles au problème explicatif posé dans le but d'accéder à la bonne solution. Nous avons, à l'aide des outils de la TAD, caractérisé les techniques de l'enseignante mobilisées dans cette mise en texte et qui semblent liées à ses conceptions concernant les savoirs scientifiques et leur construction avec les élèves. Ces techniques privilégient la construction de textes propositionnels révélant les seules solutions et favorisant la dimension assertorique à celle apodictique caractéristique des savoirs scientifiques. La production de tels textes est, rappelons-le, largement critiquée par les didacticiens car elle limite les savoirs à de simples énoncés factuels (Astolfi, 1992, 2005). Il est important de signaler que les conceptions des enseignants que nous désignons sont indirectement nourries par la «forme scolaire» des savoirs scientifiques. L'école privilégie les faits et les énoncés et c'est précisément cette forme du savoir que les enseignants transmettent à leurs élèves et qui leur a été transmise par ailleurs. Ainsi, l'institution scolaire participe dans le maintien des caractéristiques propositionnelles des savoirs scientifiques. Dans ce sens, Kahn et Rey (2008, p. 24) estiment que la forme scolaire des savoirs structure les pratiques de l'enseignant et que le repérage de cette forme et sa remise en question " permet de mieux voir certaines de ces caractéristiques et leurs conséquences sur les pratiques de l'enseignant aussi bien dans la classe qu'en dehors d'elle » (ibid.). Par ailleurs, la situation didactique ordinaire étudiée était peu propice à la problématisation même lors des moments comme les débats scientifiques censés pousser les élèves au bout de leurs explications et leurs argumentations. Qu'en est-il alors de la mise en texte lors des situations favorables à la probléma- tisation ? Orange Ravachol (2010) a caractérisé, en situation ordinaire, l'activité de deux enseignantes au cours de deux débats scientifiques en Terminale $S$ abordant le problème du magmatisme des zones de subduction. Les enseignantes concernées accordent de l'importance à la construction des raisons qui sous-tendent les solutions des problèmes et mettent en place depuis quelques temps des débats au sein de leurs séquences. De plus, elles sont engagées dans les travaux didactiques d'une équipe de recherche qui confère un rôle primordial à la problématisation. Les résultats des analyses menées par Orange Ravachol (2010) montrent une association, pour ces deux enseignantes, entre une logique de problématisation hésitante au cours du débat puis une logique de tri de la bonne solution par la preuve au terme de cette phase où les raisons discutées sont ignorées lors du passage à l'écrit. En effet, il apparaît que même si des nécessités sont construites lors du débat, elles disparaissent quand il s'agit de passer à l'étude des documents scientifiques qui servent principalement à identifier ce qui est vrai, à rejeter ce qui est faux et à repérer la bonne solution. Ainsi, lorsqu'une mise en texte orale problématisée est développée, elle se retrouve rompue par un basculement - quand débute le tri des solutions - vers une logique de réfutation mettant en avant les solutions et laissant de côté les raisons (ibid.). La logique de tri des solutions prend le devant même si la problématisation et la construction des nécessités figurent parmi les buts des enseignantes. Cette démarche va dans le sens d'une problématisation technique utilisant les raisons et les preuves empiriques pour accéder aux solutions (Orange, 2005). Or, dans une véritable problématisation scientifique (ou théorique), il s'agit, pour construire des problèmes explicatifs, de parcourir et d'examiner le champ du possible, de l'impossible et du nécessaire afin de garder la concentration sur l'apodicticité des savoirs scientifiques (ibid.). OrangeRavachol (2010) évoque la difficulté de ces enseignantes à conserver la dimension apodictique dans le texte du savoir et à l'articuler à celle assertorique. Nous remarquons que, pour l'étude de cas réalisée au cours de ce travail ainsi que celles analysées par Orange-Ravachol (2010), la priorité des enseignants est accordée aux solutions même si la construction des problèmes peut figurer parmi leurs objectifs. Cela prouve que la mise en texte problématisé est un processus complexe et difficile à mettre en place. Pour construire un savoir problématisé, il ne 
suffit pas de mener un travail de construction des problèmes pendant les débats scientifiques qui ne constituent que des moments particuliers des situations d'enseignement-apprentissage. La mise en texte problématisé des savoirs nécessite que la focalisation sur les raisons soit prolongée avec les phases ultérieures de la séquence.

\section{CONCLUSION}

Nous avons - à partir de l'analyse des textes produits au cours d'une séquence ordinaire en sciences de la Terre - tenté de modéliser, grâce à des outils empruntés à la TAD, les techniques enseignantes pour une activité de mise en texte qui visiblement ne garde pas de traces de la problématisation. Il apparaît que les difficultés d'une mise en texte problématisé sont liées à des techniques centrées sur le repérage et de tri des solutions dans le but d'identifier la bonne solution au problème posé. Ces techniques guident manifestement l'activité de l'enseignante et font qu'elle ne va pas dans le sens d'un travail de problématisation. Elles semblent justifiées par des technologies marquées par une certaine conception des savoirs scientifiques. La façon de mettre en texte le savoir scientifique que nous avons pointé constitue un obstacle à la problématisation et à la construction des savoirs problématisés. Elle fait que les élèves, comme le note Fabre (2007), accèdent à des savoirs présentés sous forme de textes exposant les résultats de la science et masquant la problématisation dont ils sont issus. Mais à quelles conditions peut-on accéder à des textes de savoirs associant les raisons aux solutions et avec? L'étude de quelles pratiques cette question nécessite des approfondissements et la poursuite de ce travail de recherche par l'analyse ou la construction d'autres séquences d'enseignement-apprentissage.

\section{NOTES}

1. Nous utiliserons les termes «sciences de la Terre » et " géologie » comme des synonymes tout au long de cette recherche.

2. La notion de «synopsis » est un élément majeur de la théorie de l'action conjointe en didactique (TACD) développée par Sensevy (2011). Thiberghien et al. (2007) travaillent dans cette théorie.

3. Nous appelons « débat " cette phase mais il faudrait déterminer, lors de nos analyses suivantes, s'il s'agit d'un véritable débat scientifique au sens de Fabre \& Orange (1997).

4. Les écrits intermédiaires « restent étroitement associés à des situations d'élaboration transitoires, soit de travail personnel, soit de travail collectif » (Chabanne $\&$ Bucheton, 2002, p. 26). Nous symbolisons ces écrits par les encadrés à traits pointillés tandis que les écrits finaux sont symbolisés par des traits pleins.

5. Ce document est repris d'un cahier d'élève.

6. La « croissance progressive du cône volcanique » figurant sur le schéma bilan a fait l'objet d'une mise en texte séparée de celle de l'origine des matériaux volcaniques que nous n'abordons pas dans cette étude.

7. Les extraits de retranscription présentés en gras permettent d'appuyer les analyses réalisées. Cette remarque est valable pour tous les extraits mentionnés dans cet article. 


\section{RÉFÉRENCES}

Allègre, C. (1987). Les fureurs de la Terre. Paris : Odile Jacob.

Astolfi, J.-P. (1992). Lécole pour apprendre. Paris : ESF.

Astolfi, J.-P. (2005). Problèmes scientifiques et pratiques de formation. In O. Maulini \& C. Montandon Les formes de l'éducation: variété et variations, p. 65-81. Raisons éducatives, Bruxelles : De Boeck.

Bachelard, G. (1949). Le rationalisme appliqué. Paris : Presses universitaires de France.

Beorchia, F. (2003). La communication nerveuse : conceptions des apprenants et problématisation. Importance des explications mécanistes et vitalistes. Thèse de doctorat, université de Nantes.

Bronckart, J.-P. (1996). Activités langagières, textes et discours. Paris : Delachaux et Niestlé.

Brossard, M. (1998). Approche socio-historique des situations d'apprentissage de l'écrit, In M. Brossard \& Fijalko, J. (Éds.), (s.d.). Apprendre à l'école : perspectives piagétiennes et vygotskiennes, (p. 37-50), Pessac: Presses universitaires de Bordeaux.

Bosch, M. \& Chevallard, Y. (1999). La sensibilité de l'activité mathématique aux ostensifs. Recherches en Didactique des Mathématiques, 19, 77-124.

Calmettes, B. (2011). Analyse pragmatique de pratiques ordinaires, rapport pragmatique à l'enseigner : étude de cas : des enseignants experts, en démarche d'investigation en physique. Recherches en didactiques des sciences et des technologies, 2, 235-272.

Chabanne, J.-C. \& Bucheton, D. (2002). Lactivité réflexive dans les écrits intermédiaires: quels indicateurs ? In J.-C. Chabanne \& D. Bucheton (Éds.), Parler et écrire pour penser, apprendre et se construire: L'écrit et l'oral réflexif,(p. 25-51). Paris: Presses universitaires de France.

Chalak, H. (2012a). Conditions didactiques et difficultés de construction de savoirs problématisés en sciences de la Terre : étude de la mise en texte des savoirs et des pratiques enseignantes dans des séquences ordinaires et forcées concernant le magmatisme (collège et lycée). Thèse de doctorat, université de Nantes.

Chalak, H. (2012b). Problématisation et construction de textes de savoirs dans le domaine du magmatisme au collège. Recherches en didactique des sciences et des technologies RDST, 6, 119-160.

Chevallard, Y. \& Joshua, M.-A. (1991). La transposition didactique : du savoir savant au savoir enseigné. La pensée sauvage.

Chevallard, Y. (1997). Familière et problématique, la figure du professeur. Recherches en Didactique des Mathématiques, 17, 17-54.

Chevallard, Y. (1998). Analyse des pratiques enseignantes et didactique des mathématiques : l'approche anthropologique. In Actes de l'université d'été, La Rochelle.

Fabre, M. (1999). Situations-problèmes et savoir scolaire. Paris : Presses universitaires de France.
Fabre, M. (2007). Des savoirs scolaires sans problèmes et sans enjeux. La faute à qui ? Revue française de pédagogie, 161, 69-78.

Fabre, M. (2009). Philosophie et pédagogie du problème. Paris : Jean Vrin.

Fabre, M. (2011). Éduquer pour un monde problématique. La carte et la boussole. Paris: Presses universitaires de France.

Fabre, M. \& Orange, C. (1997). Construction des problèmes et franchissements d'obstacles. ASTER, 24, 37-57.

Goix, H. (1995). Vous avez dit : cristal ? Je pense : verre. ASTER, 20, 105-137.

Kahn, S. \& Rey, B. (2008). Pratiques d'enseignement, forme scolaire et difficultés des élèves. Les dossiers des sciences de l'éducation, 19, 13-25.

Martinand, J.-L. (1986). Connaître et transformer la matière. Berne : Peter Lang.

M.E.N. (2008). Programmes de l'enseignement de Sciences de la vie et de la terre (collège). B.O.E.N., 6, 28 août 2008.

Mercier, A. (2008). Pour une lecture anthropologique du programme didactique. Éducation et didactique, 2, 7-40. En ligne http://educationdidactique.revues.org/251, consulté le 21 novembre 2011.

Mercier, A., Schubauer-Leoni, M. \& Sensevy, G. (2002). Vers une didactique comparée. Revue française de pédagogie, 141, 5-16.

Orange, C. (1995). Volcanisme et fonctionnement interne de la Terre : repères didactiques pour un enseignement de l'école élémentaire au lycée. ASTER, 20, 85-103.

Orange, C. (2000). Idées et raisons : construction de problèmes, débats et apprentissages scientifiques en Sciences de la vie et de la Terre. Mémoire de recherche pour l'H.D.R., université de Nantes.

Orange, C. (2003). Débat scientifique dans la classe, problématisation et argumentation: le cas d'un débat sur la nutrition au cours moyen. ASTER, 37, 83-107.

Orange, C. (2005). Problématisation et conceptualisation en sciences et dans les apprentissages scientifiques. Les sciences de l'éducation, Pour l'ère nouvelle, 38, 69-93.

Orange, C. (2006). Problématisation, savoirs et apprentissages en sciences. In M. Fabre \& E. Vellas (Éds.), Situations de formation et problématisation, 75-90. Perspectives et éducation et formation.

Orange, C. (2007). Lapprentissage scientifique ce qui se construit et ce qui se transmet. Recherches en éducation, 4, 85-92. En ligne http://www.recherches-en-education.net/IMG/pdf/REE-no4.pdf, consulté le 31 août 2011.

Orange, C. (2009). Réel, langages et apprentissages scientifiques. In P. Schneeberger \& A. Vérin (Éds.), Développer des pratiques d'oral et d'écrit en sciences. Quels enjeux pour les apprentissages à l'école, (p. 245-250). INRP.

Orange, C. \& Orange-Ravachol, D. (2004). Les conceptions des élèves et leur mode de raisonnement en sciences de la Terre. Géochronique, 90, 29-32.

Orange, C. \& Orange-Ravachol, D. (2007). Problématisation et mise en texte des savoirs scolaires : le cas d'une 
séquence sur les mouvements corporels au cycle 3 de l'école élémentaire. In Actes des $5^{\text {es }}$ rencontres scientifiques de l'ARDIST, (p. 305-312). La Grande Motte. En ligne http://ardist.aix-mrs.iufm.fr/wp-content/ actes2007, consulté le 31 août 2011.

Orange-Ravachol, D. (2003). Utilisation du temps et explications en Sciences de la Terre par les élèves de lycée: étude de quelques problèmes géologiques. Thèse de doctorat, université de Nantes.

Orange-Ravachol, D. (2010). Collaboration chercheur didacticien / enseignant et choix de l'enseignant en situation scolaire: une étude de cas en sciences de la Terre. Recherches en éducation, H-S. 1, 47-59. En ligne http:// www.recherches-en-education.net, consulté le 6 septembre 2011.

Popper, K. (1991). La connaissance objective. Paris : Aubier.

Reboul, O. (1992). Les valeurs de l'éducation. Paris : Presses universitaires de France.

Rey, B. (2005). Peut-on enseigner la problématisation? Recherche et Formation, 48, 91-105.

Rey, B. (2007). Pourquoi l'école s'obstine-t-elle à vouloir faire acquérir des savoirs ? In M. Durand, M. Fabre, M. Antoine \& Maulini, O. (Éds.), Les situations de formations entre savoirs, problèmes et activités, (p. 171-190). L'harmattan.

Santini, J. (2009). Les conceptions des élèves : des données empiriques aux résultats inférés. Questionner l'implicite d'un objet des didactiques à partir des conceptions du mécanisme sismique. In C. Cohen-Azria \&
N. Sayac (Éds.), Questionner l'implicite. Les méthodes de recherche en didactiques, (p. 227-242). Villeneuve d'Ascq : Presses universitaires du Septentrion.

Santini, J. (2010). Les systèmes sémiotiques dans l'action conjointe en didactique. Une étude de cas en géologie à l'école élémentaire: la coupe d'un appareil volcanique. Recherches en didactique des sciences et des technologies RDST, 2, 159-191.

Sensevy, G. (2011). Le Sens du Savoir. Éléments pour une Théorie de l'Action Conjointe en Didactique. Bruxelles: De Boeck.

Schubauer-Leoni, M.-L. \& Leutenegger, F. (2002). Expliquer et comprendre dans une approche clinique/expérimentale du didactique ordinaire. In F. Leutenegger \& M. Saada-Robert (Éds.), Expliquer, comprendre en sciences de l'éducation, (p. 227-251). Bruxelles : De Boeck.

Tiberghien, A., Malkoun, L., Buty, C., Souassy, N. \& Mortimer, E. (2007). Analyse des savoirs en jeu en classe de physique à différentes échelles de temps. In G. Sensevy \& A. Mercier (Éds.), Agir ensemble. L'action didactique conjointe du professeur et des élèves, (p. 93122). Rennes: Presses universitaires de Rennes.

Venturini, P., Calmettes, B., Amade-Escot, C. \& Terrisse, A. (2007). Analyse didactique des pratiques d'enseignement de la physique d'une professeure expérimentée. ASTER, 45, 211-233.

Verret, M. (1975). Le temps des études. Paris : Librairie Honoré Champion. 


\section{ANNEXE 1.}

Document scientifique du manuel scolaire exploité par la classe de $4^{\mathrm{e}}$ - séquence ordinaire

\section{Le " moteur n des eruptions volcaniques}

- Les éruptions

volcaniques

observables

en surface résultent

de phénoménes

profonds.

Un réservoir de

magma ou réservoir

magmatique

en profondeur.

\section{L'erregstrement}

de l'activite sisnique

diun volcan permet

de localiser

en protondeur

des foyees sisnipues.

Des ondes sisnisues

se propageant peu

dans les liquildss.

l'absence ici de foyers

a la verticelo

du valcan sugitre

Fexistence drune rone

(icl, on gris)

coestifinte

de natb́riexax fluideg:

le réservoir de nagna

situr en protondeur.

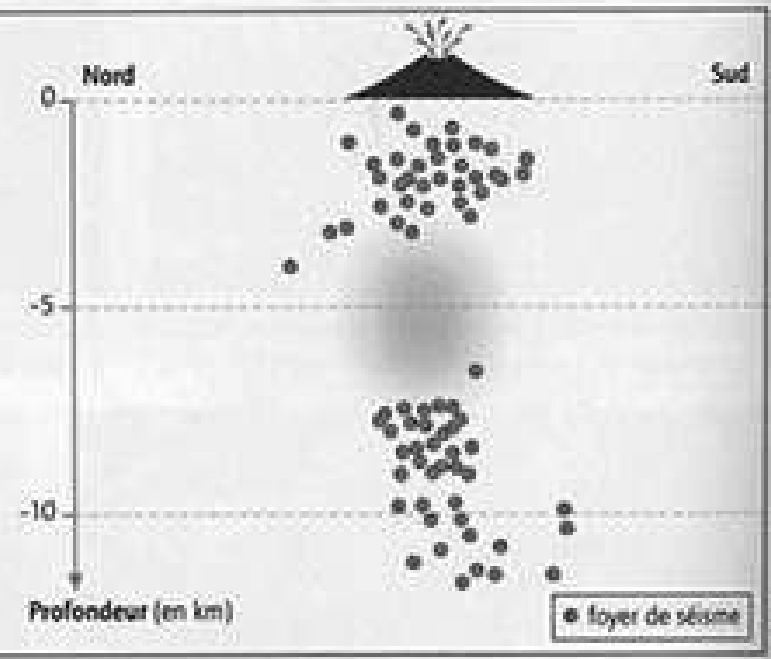

Le moteur des éruptions volcaniques (Éditions Belin, SVT 4e, 2007, p. 158). 